\title{
Bayesian Deep Net GLM and GLMM
}

\author{
M.-N. Tran* N. Nguyen* $\quad$ D. Nott ${ }^{\dagger} \quad$ R. Kohn ${ }^{\ddagger}$
}

\begin{abstract}
Deep feedforward neural networks (DFNNs) are a powerful tool for functional approximation. We describe flexible versions of generalized linear and generalized linear mixed models incorporating basis functions formed by a DFNN. The consideration of neural networks with random effects is not widely used in the literature, perhaps because of the computational challenges of incorporating subject specific parameters into already complex models. Efficient computational methods for high-dimensional Bayesian inference are developed using Gaussian variational approximation, with a parsimonious but flexible factor parametrization of the covariance matrix. We implement natural gradient methods for the optimization, exploiting the factor structure of the variational covariance matrix in computation of the natural gradient. Our flexible DFNN models and Bayesian inference approach lead to a regression and classification method that has a high prediction accuracy, and is able to quantify the prediction uncertainty in a principled and convenient way. We also describe how to perform variable selection in our deep learning method. The proposed methods are illustrated in a wide range of simulated and real-data examples, and the results compare favourably to a state of the art flexible regression and classification method in the statistical literature, the Bayesian additive regression trees (BART) method. User-friendly software packages in Matlab, R and Python implementing the proposed methods are available at https://github.com/VBayesLab.
\end{abstract}

Keywords. Deep learning; Factor models; Reparametrization gradient; Stochastic optimization; Variational approximation; Variable selection.

\section{Introduction}

Deep feedforward neural network (DFNN) modeling provides a powerful technique for approximating regression functions with multiple regressors, and has become increasingly popular recently. DFNNs have been applied successfully in fields such as

* Discipline of Business Analytics, The University of Sydney Business School and ACEMS.

$\dagger$ Department of Statistics and Applied Probability, National University of Singapore.

${ }^{\ddagger}$ School of Economics, UNSW Business School and ACEMS. 
image processing, computer vision and language recognition. See Schmidhuber (2015) for a historical survey and Goodfellow et al. (2016) for a more comprehensive recent discussion of DFNNs and other types of neural networks, collectively known as deep learning models.

This paper considers variants of generalized linear models (GLMs) and generalized linear mixed model (GLMMs) using DFNNs as a way to efficiently transform a vector of $p$ raw covariates $X=\left(X_{1}, \ldots, X_{p}\right)^{\top}$ into a new vector of $m$ predictors $Z$ in the model. We refer to these DFNN-based versions of GLM and GLMM as DeepGLM and DeepGLMM, respectively. A conventional GLM uses a link function that links the conditional mean of the response variable $Y$ to a linear combination of the predictors $Z=\phi(X)=\left(\phi_{1}(X), \ldots, \phi_{m}(X)\right)^{\top}$, with each $\phi_{j}(X)$ a function of $X$. We refer to the original raw input variables $X_{j}$ as covariates, and refer to the transformations $\phi_{j}(X)$ as predictors. In conventional GLMs, the $\phi_{j}(X)$ are chosen a priori in some way before any model selection, but here we are concerned with learning an appropriate $Z$ from data through a flexible smooth transformation. In the machine learning literature the predictors $Z$ are commonly referred to as learned features. If a DFNN is used for transforming the covariates, then $Z$ has the form

$$
Z=f_{L}\left(W_{L}, f_{L-1}\left(W_{L-1}, \cdots f_{1}\left(W_{1}, X\right) \cdots\right)\right),
$$

which is often graphically represented by a network as in Figure 1. Each vector-

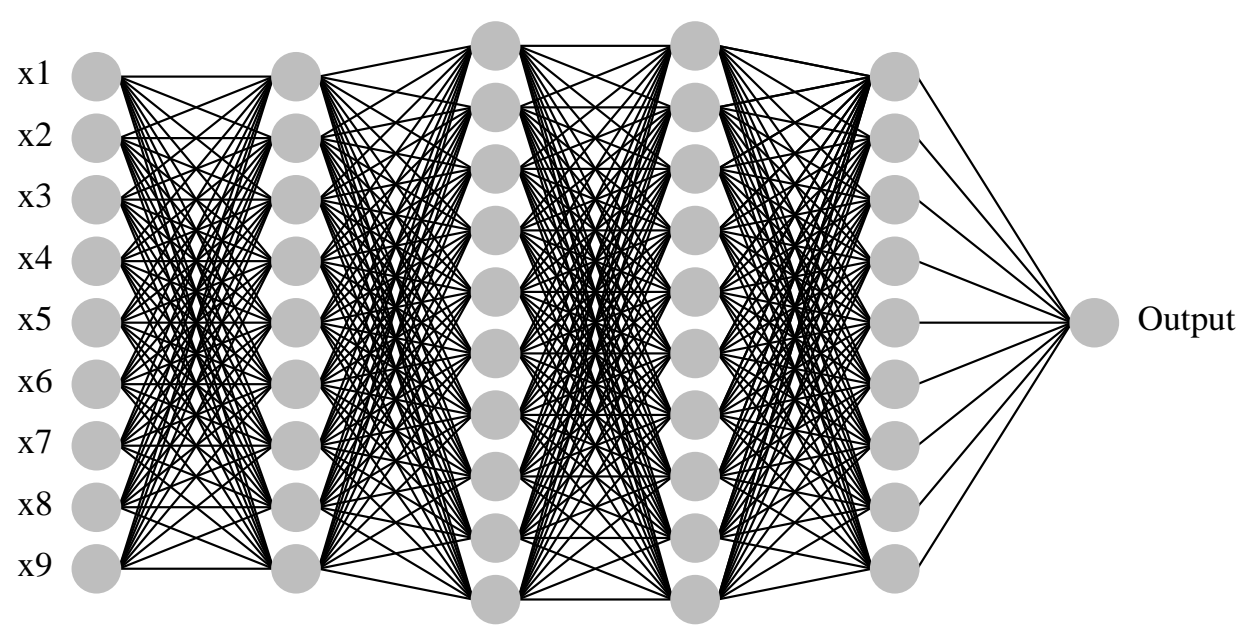

Figure 1: Graphical representation of a layered composition function with $L=4$ hidden layers. The input layer represents 9 raw covariates $X$. The last hidden layer (hidden layer 4) represents the predictors $Z$.

valued function $Z_{l}=f_{l}\left(W_{l}, Z_{l-1}\right), l=1,2, \ldots, L$, is called a hidden layer, $L$ is the number 
of hidden layers in the network, $w=\left(W_{1}, \ldots, W_{L}\right)$ is the set of weights, and we define $Z_{0}=X$. The function $f_{l}\left(W_{l}, Z_{l-1}\right)$ is assumed to be of the form $h_{l}\left(W_{l} Z_{l-1}\right)$, where $W_{l}$ is a matrix of weights that connect layer $l$ to layer $l+1$ and $h_{l}(\cdot)$ is a scalar function, called the activation function. Applying $h_{l}$ to a vector should be understood component-wise. For a discussion of alternative kinds of architectures for deep neural networks see, for example, Goodfellow et al. (2016). The architecture (1) provides a powerful way to transform the raw covariate data $X$ into summary statistics $Z$ that have some desirable properties. In the DeepGLM, we link the conditional mean of the response $\xi=\mathbb{E}(Y \mid X)$ to a linear combination of $Z$

$$
g(\xi)=\beta_{0}+Z^{\top} \widetilde{\beta}
$$

and the model parameters consist of $w, \beta$ and other possible parameters such as any dispersion parameters. Section 2.2 defines DeepGLMM models by adding random effects to DeepGLM models. Such models are designed to model within-subject dependence. The use of neural network basis functions in the context of mixed effects models seems not considered much in the literature. Lai et al. (2006) is the only work that we know of that deals with a neural network basis and random effects, and they use it for modeling pharmacokinetic data. They consider neural networks with only one hidden layer, however.

Estimation and variable selection in complex and high-dimensional models like DeepGLM and DeepGLMM are challenging. This article develops Bayesian inference based on variational approximation, which provides an approach to approximate Bayesian inference that is useful for many modern applications involving complex models and large datasets. We also consider variable selection, which is often of primary interest in statistics but is somewhat overlooked in the deep learning literature. We describe a Bayesian adaptive group lasso method (Leng et al., 2014; Kyung et al., 2010) for variable selection in DeepGLM and DeepGLMM, in which the adaptive shrinkage parameters are estimated using marginal likelihood maximization with the optimization updating procedure conveniently embeded within the variational approximation. Our variational approximation scheme assumes a multivariate Gaussian approximating family. With such a family, parsimonious but flexible methods for parametrizing the covariance matrix are necessary if the approach is to be useful for problems with a high-dimensional parameter. Here we consider factor parametrizations (Bartholomew et al., 2011) which are often effective for describing dependence in high dimensional settings. We discuss efficient methods for performing the variational optimization in this context using the natural gradient (Amari, 1998) by leveraging the factor structure and using iterative conjugate gradient methods for solving large linear systems. In the case with a one-factor decomposition, we show that the natural gradient can be computed efficiently without iterative conjugate gradient methods, which leads to a particularly simple Gaussian variational method for fitting high-dimensional models that can often be adequate for predictive inference. We will refer to this estimation method as the NAtural gradient Gaussian Variational 
Approximation with factor Covariance (NAGVAC).

We illustrate the DeepGLM and DeepGLMM and the training method NAGVAC in a range of experimental studies and applications, with a focus on datasets of only moderate size where quantification of prediction uncertainty is important. The Bayesian approach we follow is attractive in these applications as it allows a principled and automatic way for selecting the (many) shrinkage parameters; see Section 4. The Bayesian approach also leads to a principled and convenient way for quantifying prediction uncertainty through prediction intervals on test data, which would be challenging to do so with non-Bayesian approaches for large models like DeepGLM. Many successful applications of deep learning are in image processing and speech recognition, where the datasets are large and have some special domain-application characteristics such as association between the local pixels in an image. Skepticism has sometimes been expressed about whether deep learning methods are useful for applications involving limited data where both prediction accuracy and quantifying prediction uncertainty are the focus. The main conclusion in our examples is that the DFNN-based regression models DeepGLM and DeepGLMM perform very well in terms of prediction accuracy and prediction uncertainty, provided appropriate attention is paid to regularization methods, prior distributions and computational algorithms. We obtain results which compare favourably to the Bayesian Additive Regression Trees method (BART) of Chipman et al. (2010), which is a commonlyused flexible regression and classification method in the statistics literature. Software packages in Matlab, $\mathrm{R}$ and Python implementing the proposed methods are available at https://github.com/vbayeslab.

Related work. Polson and Sokolov (2017) provide a Bayesian perspective on DFNN methodology and explain its interesting connection to statistical techniques such as principal component analysis and reduced rank regression. However, they do not provide any training method, which is a challenging problem in Bayesian inference with DFNN.

The NAGVAC method is closely related to Ong et al. (2017a), who consider Gaussian variational approximation with a factor covariance structure using stochastic gradient approaches for the optimization. Their approach uses the so-called reparametrization trick (Kingma and Welling, 2013, Rezende et al., 2014) and its modification by Roeder et al. (2017) for estimating gradients of the variational objective. However, for certain models where it is very challenging to optimize the variational objective, first-order optimization methods such as those considered in Ong et al. (2017a) may be very slow to converge. Ong et al. (2017b) consider natural gradient methods for Gaussian variational approximations using a factor covariance structure. However, their work was in the context of likelihood-free inference methods with a parameter of dimension at most a few hundred, and the approach they develop does not scale to larger problems. The current work shows how the natural gradient Gaussian variational approximation with factor covariance can be implemented in very high dimensions. 
Much of the recent literature relevant to our training method occurs in the field of deep learning. Martens (2010) considers second-order optimization methods in deep learning and describes Hessian-free optimization methods, adapting a long history of related methods in the numerical analysis literature to that context. A detailed discussion of the connections between natural gradient methods and second order optimization methods is given in Martens (2014). Pascanu and Bengio (2014) consider the use of the natural gradient in deep learning problems and its connections with Hessian-free optimization, but like Martens (2010) their work is not specifically concerned with variational objectives. Fan et al. (2015) consider how to implement Hessian-free methods for the case of a variational objective function and Gaussian approximating family. Similarly to our development here, they consider using conjugate gradient linear solvers as an efficient solution to the difficult matrix calculations that occur in a naive formulation of second-order methods. By a reparametrization approach they are able to obtain estimates of Hessian vector products. They do not consider factor parametrizations of the covariance structure, however. Here we leverage the factor covariance structure to calculate the matrix vector products we need directly, without the need to store large matrices. Recently Regier et al. (2017) consider a second order trust region method for black box variational inference. They show that while their approach may be more expensive per iteration than common first-order methods for variational Gaussian approximation such as those implemented in Titsias and Lázaro-Gredilla (2014) and Kucukelbir et al. (2017), it reduces total computation time and provably converges to a stationary point. It may be useful to consider how to exploit a factor parametrization of the covariance structure in the framework they develop, but this is not considered here.

The next section describes the two new classes of flexible statistical models DeepGLM and DeepGLMM. Section 3 describes the natural gradient Gaussian variational approximation method. Section 4 describes the variable selection method and highlights advantages of our fully Bayesian treatment. Section 5 presents practical recommendations in training. Section 6 presents experimental studies and applications, and Section 7 concludes. The Appendix gives details of the natural gradient computation and more details of an example.

\section{Flexible regression models with DFNN}

This section presents the DeepGLM and DeepGLMM models. Deep feedforward neural network models, or multi-layer perceptrons, for classification and regression with a continuous response have been widely used in the machine learning literature. We use statistical terminologies and unify these models under the popular GLM framework, and propose the DFNN-based version of GLMM for analyzing panel data. 


\subsection{DeepGLM}

Consider a dataset $D=\left\{\left(y_{i}, x_{i}\right), i=1, \ldots, n\right\}$ with $y_{i}$ the response and $x_{i}=\left(x_{i 1}, \ldots, x_{i p}\right)^{\top}$ the vector of $p$ covariates. We also use $y$ and $x$ to denote a generic response and a covariate vector, respectively. Consider a neural net with the input vector $x$ and a scalar output as represented in Figure 1. Denote by $z_{j}=\phi_{j}(x, w), j=1, \ldots, m$, the units in the last hidden layer, where $w$ is the weights up to the last hidden layer, and $\beta=\left(\beta_{0}, \beta_{1}, \ldots, \beta_{m}\right)^{\top}$ are the weights that connect the $z_{j}$ to the output which we write as

$$
\mathfrak{N}(x, w, \beta)=\beta_{0}+\beta_{1} z_{1}+\ldots+\beta_{m} z_{m}=\beta_{0}+\widetilde{\beta}^{\top} z
$$

with $\widetilde{\beta}=\left(\beta_{1}, \ldots, \beta_{m}\right)^{\top}$ and $z=\left(z_{1}, \ldots, z_{m}\right)^{\top}$.

We assume that the conditional density $p(y \mid x)$ has an exponential family form

$$
p(y \mid x)=\exp \left(\frac{y \varpi-b(\varpi)}{\phi}+c(\phi, y)\right)
$$

with the canonical parameter $\varpi$ and the dispersion parameter $\phi$. Let $g(\cdot)$ be the link function that links the conditional mean $\xi=\xi(x)=\mathbb{E}(y \mid x)$ to a function of the covariates $x$. In the conventional GLM, $g(\xi)$ is assumed to be a linear combination of $x$. In order to achieve flexibility and capture possible non-linear effects that $x$ has on $\xi$, we propose the more flexible model

$$
g(\xi)=\beta_{0}+\widetilde{\beta}^{\top} z=\mathfrak{N}(x, w, \beta) .
$$

In our later examples we use the canonical link function where $\varpi=g(\xi)$, which is the $\log$ link for Poisson responses and the logit link for binomial responses. The predictors (learned features) $z_{j}$ efficiently capture the important non-linear effects of the original raw covariates $x$ on $g(\xi)$.

The model (3) is flexible, but can be hard to interpret. It may be useful in this respect to introduce additional structure. One possibility is to partition the covariates as $x=\left(x^{(1)^{\top}}, x^{(2)^{\top}}\right)^{\top}$ with $x^{(1)}$ and $x^{(2)}$ expected to have nonlinear and linear effects respectively on $g(\xi)$, so that

$$
g(\xi)=\mathfrak{N}\left(x^{(1)}, w, \beta^{(1)}\right)+\beta^{(2)^{\top}} x^{(2)}
$$

where $\beta^{(1)}$ parametrizes the nonlinear effects w.r.t. the covariates $x^{(1)}$ and $\beta^{(2)}$ parametrizes the linear effects w.r.t. the covariates $x^{(2)}$. Write $\beta=\left(\beta^{(1)}, \beta^{(2)}\right)$. We refer to the general regression model with the exponential family for response distribution (2) and the mean model (3) or (4) as DeepGLM.

The vector of model parameters $\theta$ consists of $w, \beta$ and possibly dispersion parameters $\phi$ if $\phi$ is unknown. The density $p(y \mid x)$ in (2) is now a function of $\theta$, $p(y \mid x)=p(y \mid x, \theta)$. Given a dataset $D$, the likelihood function is

$$
L(\theta)=\prod_{i=1}^{n} p\left(y_{i} \mid x_{i}, \theta\right)
$$

and likelihood-based inference methods, including Bayesian methods, can be applied. 


\subsection{DeepGLMM}

Consider a panel dataset $D=\left\{\left(y_{i t}, x_{i t}\right), t=1, \ldots, T_{i}, i=1, \ldots, n\right\}$ with $y_{i t}$ the response and $x_{i t}$ the vector of covariates of subject $i$ at time $t$. Generalized linear mixed models (GLMM) use random effects to account for within-subject dependence. Let $z_{i t, j}=\phi_{j}\left(x_{i t}, w\right), j=1, \ldots, m$, be the units in the last hidden layer of a neural net, $z_{i t}=$ $\left(z_{i t, 1}, \ldots, z_{i t, m}\right)^{\top}$. Similarly to GLMM, to account for within-subject dependence, we propose to link the conditional mean of $y_{i t}$ given $x_{i t}, \xi_{i t}=\mathbb{E}\left(y_{i t} \mid x_{i t}\right)$, to the predictors $z_{i t, j}$ as follows

$$
g\left(\xi_{i t}\right)=\beta_{0}+\alpha_{i 0}+\left(\beta_{1}+\alpha_{i 1}\right) z_{i t, 1}+\ldots+\left(\beta_{m}+\alpha_{i m}\right) z_{i t, m}=\mathfrak{N}\left(x_{i t}, w, \beta+\alpha_{i}\right),
$$

where $\alpha_{i}=\left(\alpha_{i 0}, \ldots, \alpha_{i m}\right)^{\top}$ are random effects that reflect the characteristics of subject $i$. The variation between the subjects is captured in the distribution of $\alpha_{i}$. Our paper assumes $\alpha_{i} \sim \mathcal{N}(0, \Gamma)$ but more flexible distributional specifications for the random effects can also be considered.

Similarly to the previous section, a more interpretable model can be developed if some additional structure is assumed. Partition the covariates $x_{i t}$ as $\left(x_{i t}^{(1)^{\top}}, x_{i t}^{(2)^{\top}}\right)^{\top}$ where $x_{i t}^{(1)}$ and $x_{i t}^{(2)}$ are expected to have non-linear and linear effects respectively on $g\left(\xi_{i t}\right)$ :

$$
g\left(\xi_{i t}\right)=\mathfrak{N}\left(x_{i t}^{(1)}, w, \beta^{(1)}\right)+\left(\alpha_{i}+\beta^{(2)}\right)^{\top} x_{i t}^{(2)},
$$

where $\beta^{(1)}$ parametrizes fixed non-linear effects for $x_{i t}^{(1)}$, and $\beta^{(2)}$ and $\alpha_{i}$ are fixed and random linear effects w.r.t. the covariates $x_{i t}^{(2)}$. Write $\beta=\left(\beta^{(1)}, \beta^{(2)}\right)$. The model parameters $\theta$ include $w, \beta, \Gamma$ and any dispersion parameters $\phi$ if unknown. We refer to the panel data model with the distribution (2) and the link (6) or (7) as DeepGLMM.

The likelihood for the DeepGLMM is

$$
L(\theta)=\prod_{i=1}^{n} L_{i}(\theta)
$$

with the $i$ th likelihood contribution

$$
\begin{aligned}
L_{i}(\theta)=p\left(y_{i} \mid x_{i}, \theta\right) & =\int p\left(y_{i} \mid x_{i}, w, \beta, \phi, \alpha_{i}\right) p\left(\alpha_{i} \mid \Gamma\right) d \alpha_{i} \\
& =\int \prod_{t=1}^{T_{i}} p\left(y_{i t} \mid z_{i t}, \beta, \phi, \alpha_{i}\right) p\left(\alpha_{i} \mid \Gamma\right) d \alpha_{i} .
\end{aligned}
$$

Section 3 describes a VB algorithm for fitting the DeepGLMM.

The likelihood for the DeepGLMM described in (8) and (9) is intractable, because the integral in (9) cannot be computed analytically, except for the case where the conditional distribution of $y_{i t}$ given $z_{i t}$ is normal. However, we can estimate each 
likelihood contribution $L_{i}(\theta)$ unbiasedly using importance sampling, and this allows estimation methods for intractable likelihoods, such as the block pseudo-marginal MCMC of Tran et al. (2016a) or the VB method of Tran et al. (2017), to be used. We consider here an alternative VB method based on the reparametrization trick in Section 3.1, which utilizes the information of the gradient of the log-likelihood computed by the back-propagation algorithm (Goodfellow et al., 2016). By Fisher's identity (Gunawan et al., 2017), the gradient of the log-likelihood contribution $\nabla_{\theta} \ell_{i}(\theta)$, $\ell_{i}(\theta)=\log L_{i}(\theta)$ with $L_{i}(\theta)$ in $(9)$, is

$$
\nabla_{\theta} \ell_{i}(\theta)=\int \nabla_{\theta}\left(\log \prod_{t=i}^{T_{i}} p\left(y_{i t} \mid z_{i t}, \beta, \phi, \alpha_{i}\right) p\left(\alpha_{i} \mid \Gamma\right)\right) p\left(\alpha_{i} \mid \theta, y_{i}, x_{i}\right) d \alpha_{i},
$$

where $p\left(\alpha_{i} \mid \theta, y_{i}, x_{i}\right)$ is the conditional distribution of the random effects $\alpha_{i}$ given data $\left(y_{i}, x_{i}\right)$ and $\theta$. The gradient inside the integral (10) can be computed by backpropagation, and then the integral can be estimated easily by importance sampling.

\section{Gaussian variational approximation with factor covariance structure}

This section describes the NAtural gradient Gaussian Variational Approximation with factor Covariance method (NAGVAC) for approximate Bayesian inference in highdimensional models. We note that this estimation method can be used for training other high-dimensional models rather than the DeepGLM and DeepGLMM described in Section 2. Let $D$ be the data and $\theta \in \Theta$ the vector of unknown parameters. Bayesian inference about $\theta$ is based on the posterior distribution with density function

$$
\pi(\theta)=p(\theta \mid D)=\frac{p(\theta) L(\theta)}{p(D)}
$$

with $p(\theta)$ the prior, $L(\theta)=p(D \mid \theta)$ the likelihood function and $p(D)=\int p(\theta) L(\theta) d \theta$ the marginal likelihood. In all but a few simple cases the posterior $\pi(\theta)$ is unknown, partly because $p(D)$ is unknown, which makes it challenging to carry out Bayesian inference.

In this work, we are interested in variational approximation methods, which are widely used as a scalable and computationally effective method for Bayesian computation (Bishop, 2006: Blei et al., 2017). We will approximate the posterior $\pi(\theta)$ by a Gaussian distribution with density $q_{\lambda}(\theta)=\mathcal{N}(\theta ; \mu, \Sigma)$, the density of a multivariate normal distribution with mean vector $\mu$ and covariance matrix $\Sigma$. The optimal variational parameter $\lambda=(\mu, \Sigma)$ is chosen by minimizing the Kullback-Leibler divergence between $q_{\lambda}(\theta)$ and $\pi(\theta)$

$$
\begin{aligned}
\mathrm{KL}(\lambda) & =\int q_{\lambda}(\theta) \log \frac{q_{\lambda}(\theta)}{\pi(\theta)} d \theta=\int q_{\lambda}(\theta) \log \frac{q_{\lambda}(\theta)}{p(\theta) L(\theta)} d \theta+\log p(D) \\
& =-\mathrm{LB}(\lambda)+\log p(D),
\end{aligned}
$$


where

$$
\mathrm{LB}(\lambda)=\int q_{\lambda}(\theta) \log \frac{p(\theta) L(\theta)}{q_{\lambda}(\theta)} d \theta
$$

is a lower bound on $\log p(D)$. Minimizing $\mathrm{KL}(\lambda)$ is therefore equivalent to maximizing the lower bound $\operatorname{LB}(\lambda)$. If we can obtain an unbiased estimator $\widehat{\nabla_{\lambda} \widehat{\operatorname{LB}}(\lambda)}$ of the gradient of the lower bound, then we can use stochastic optimization to maximize $\mathrm{LB}(\lambda)$, as in Algorithm 1 below.

Algorithm 1. - Initialize $\lambda^{(0)}$ and stop the following iteration if the stopping criterion is met.

- For $t=0,1, \ldots$, compute $\lambda^{(t+1)}=\lambda^{(t)}+a_{t} \widehat{\nabla_{\lambda} \mathrm{LB}}\left(\lambda^{(t)}\right)$.

The learning rate sequence $\left\{a_{t}\right\}$ in Algorithm 1 should satisfy the Robbins-Monro conditions, $a_{t}>0, \sum_{t} a_{t}=\infty$ and $\sum_{t} a_{t}^{2}<\infty$ (Robbins and Monro, 1951). The choice of $a_{t}$ is discussed later on in some detail.

\subsection{Reparametrization trick}

As is typical of stochastic optimization algorithms, the performance of Algorithm 1 depends greatly on the variance of the noisy gradient so that variance reduction methods are needed. We will use the so-called reparametrization trick (Kingma and Welling, 2013; Rezende et al., 2014) in this paper, and its modification by Roeder et al. (2017), who generalized ideas considered in Han et al. (2016) and Tan and Nott (2017).

Suppose that for $\theta \sim q_{\lambda}(\cdot)$, there exists a deterministic function $g(\lambda, \epsilon)$ such that $\theta=g(\lambda, \epsilon) \sim q_{\lambda}(\theta)$ where $\epsilon \sim p_{\epsilon}(\cdot)$, which is independent of $\lambda$. For example, if $q_{\lambda}(\theta)=$ $\mathcal{N}(\theta ; \mu, \Sigma)$ then $\theta=\mu+\Sigma^{1 / 2} \epsilon$ with $\epsilon \sim \mathcal{N}(0, I)$ and $I$ is the identity matrix. Writing $\mathrm{LB}(\lambda)$ as an expectation with respect to $p_{\epsilon}(\cdot)$ gives

$$
\operatorname{LB}(\lambda)=\mathbb{E}_{\epsilon}\left(h(g(\epsilon, \lambda))-\log q_{\lambda}(g(\epsilon, \lambda))\right)
$$

where $\mathbb{E}_{\epsilon}(\cdot)$ denotes expectation with respect to $p_{\epsilon}(\cdot), h(\theta):=\log (p(\theta) L(\theta))$. Differentiating under the integral sign and simplifying as in Roeder et al. (2017) gives

$$
\nabla_{\lambda} \mathrm{LB}(\lambda)=\mathbb{E}_{\epsilon}\left(\nabla_{\lambda} g(\lambda, \epsilon) \nabla_{\theta}\left\{h(g(\epsilon, \lambda))-\log q_{\lambda}(g(\epsilon, \lambda))\right\}\right) .
$$

The gradient (13) can be estimated unbiasedly using i.i.d samples $\epsilon_{s} \sim p_{\epsilon}(\cdot), s=1, \ldots, S$, as

$$
\widehat{\nabla_{\lambda} \mathrm{LB}}(\lambda)=\frac{1}{S} \sum_{s=1}^{S} \nabla_{\lambda} g\left(\lambda, \epsilon_{s}\right) \nabla_{\theta}\left\{h\left(g\left(\lambda, \epsilon_{s}\right)\right)-\log q_{\lambda}\left(g\left(\lambda, \epsilon_{s}\right)\right)\right\} .
$$


The gradient estimator (14) has the advantage that if the variational family is rich enough to contain the exact posterior, so that $\exp (h(\theta)) \propto q_{\lambda}(\theta)$ at the optimal $\lambda$, then the estimator (14) is exactly zero at this optimal value even for $S=1$ where we use just a single Monte Carlo sample from $p_{\epsilon}(\epsilon)$. Reparametrized gradient estimators are often more efficient than alternative approaches to estimating the lower bound gradient, partly because they take into account information from $\nabla_{\theta} h(\theta)$. For further discussion we refer the reader to Roeder et al. (2017).

\subsection{Natural gradient}

It is well-known that the ordinary gradient $\nabla_{\lambda} \mathrm{LB}(\lambda)$ does not adequately capture the geometry of the approximating family $q_{\lambda}(\theta)$ (Amari, 1998). A small Euclidean distance between $\lambda$ and $\lambda^{\prime}$ does not necessarily mean a small Kullback-Leibler divergence between $q_{\lambda}(\theta)$ and $q_{\lambda^{\prime}}(\theta)$. Rao $(1945)$ was the first to point out the importance of information on the geometry of the manifold of a statistical model and introduced the Riemannian metric on this manifold induced by the Fisher information matrix. Amari (1998) shows that the steepest direction for optimizing the objective function $\operatorname{LB}(\lambda)$ on the manifold formed by the family $q_{\lambda}(\theta)$ is directed by the so-called natural gradient which is defined by pre-multiplying the ordinary gradient with the inverse of the Fisher information matrix

$$
\nabla_{\lambda} \operatorname{LB}(\lambda)^{\text {nat }}=I_{F}^{-1}(\lambda) \nabla_{\lambda} \operatorname{LB}(\lambda)
$$

with $I_{F}(\lambda)=\operatorname{cov}_{q_{\lambda}}\left(\nabla_{\lambda} \log q_{\lambda}(\theta)\right)$.

The use of the natural gradient in VB algorithms is considered, among others, by Sato (2001), Honkela et al. (2010), Hoffman et al. (2013), Salimans and Knowles (2013) and Tran et al. (2017). A simple demonstration of the importance of the natural gradient can be found in Tran et al. (2017). The use of the natural gradient in deep learning problems is considered in Pascanu and Bengio (2014), who show the connection between natural gradient descent and other second-order optimization methods such as Hessian-free optimization.

The main difficulty of using the natural gradient is the computation of $I_{F}(\lambda)$, and the solution of linear systems involving this matrix, which is required to compute (15). The problem is more severe in high dimensional models because this matrix often has a large size. Some approximation methods, such as the truncated Newton approach, are needed (Pascanu and Bengio, 2014). We consider in the next section an efficient method for computing $I_{F}(\lambda)^{-1} \nabla_{\lambda} \mathrm{LB}(\lambda)$ based on the use of iterative conjugate gradient methods for solving linear systems when the covariance matrix of the Gaussian variational approximation is parametrized by a factor model. We compute (15) by solving the linear system $I_{F}(\lambda) x=\nabla_{\lambda} \mathrm{LB}(\lambda)$ for $x$ using only matrix-vector products involving $I_{F}(\lambda)$, where the matrix vector products can be done efficiently both in terms of computation time and memory requirements by using the factor 
structure of the variational covariance matrix. In the special cases of one factor the natural gradient in (15) can be computed analytically and efficiently.

\subsection{Gaussian variational approximation with factor covari- ance}

We now describe in detail the Gaussian variational approximation with factor covariance (VAFC) method of Ong et al. (2017a). The VAFC method considers the multivariate normal variational family $q_{\lambda}(\theta)=\mathcal{N}(\mu, \Sigma)$, where $\Sigma$ is parametrized as

$$
\Sigma=B B^{\top}+D^{2}
$$

The factor loading matrix $B$ is of size $d \times f$, where $d$ is the dimension of $\theta$ and $f$ the number of factors, $f \ll d$, and $D$ is diagonal with diagonal entries $c=\left(c_{1}, \ldots, c_{d}\right)^{\top}$. $c$ is a vector of idiosyncratic noise standard deviations. Factor structures are well known to provide useful parsimonious representations of dependence in high-dimensional settings (Bartholomew et al., 2011). We assume $B$ is lower triangular, i.e., $B_{i j}=0$ for $j>i$. Although imposing the constraint $B_{i i}>0$ makes the factor representation identifiable (Geweke and Zhou, 1996), we do not impose this constraint to simplify the optimization. The variational optimization simply locks onto one of the equivalent modes. An intuitive generative representation of the factor structure that is the basis of our application of the reparametrization trick is the following: if we consider $\theta \sim q_{\lambda}(\theta)=\mathcal{N}\left(\mu, B B^{\top}+D^{2}\right)$, then we can represent $\theta$ as $\theta=\mu+B \epsilon_{1}+c \circ \epsilon_{2}$ where $\epsilon=\left(\epsilon_{1}^{\top}, \epsilon_{2}^{\top}\right)^{\top} \sim \mathcal{N}(0, I), \epsilon_{1}$ and $\epsilon_{2}$ have dimensions $f$ and $d$ respectively, and $\circ$ denotes the Hadamard (element by element) product for two matrices of the same size. We can see from this representation that the latent variables $\epsilon_{1}$ (the "factors", which are low-dimensional) explain all the correlation between the components, whereas component-specific idiosyncratic variance is being captured through $\epsilon_{2}$.

The variational parameters are $\lambda=\left(\mu^{\top}, \operatorname{vec}(B)^{\top}, c^{\top}\right)^{\top}$, where we have written $\operatorname{vec}(B)$ for the vectorization of $B$ obtained by stacking its columns from left to right. Ong et al. (2017a) show that the gradient of the lower bound takes the form

$$
\begin{aligned}
& \nabla_{\mu} \mathrm{LB}(\lambda)=\mathbb{E}_{\epsilon}\left(\nabla_{\theta} h\left(\mu+B \epsilon_{1}+c \circ \epsilon_{2}\right)+\left(B B^{\top}+D^{2}\right)^{-1}\left(B \epsilon_{1}+c \circ \epsilon_{2}\right)\right), \\
& \nabla_{B} \operatorname{LB}(\lambda)=\mathbb{E}_{\epsilon}\left(\nabla_{\theta} h\left(\mu+B \epsilon_{1}+c \circ \epsilon_{2}\right) \epsilon_{1}^{\top}+\left(B B^{\top}+D^{2}\right)^{-1}\left(B \epsilon_{1}+c \circ \epsilon_{2}\right) \epsilon_{1}^{\top}\right),
\end{aligned}
$$

and

$$
\nabla_{c} \mathrm{LB}(\lambda)=\mathbb{E}_{\epsilon}\left(\operatorname{diag}\left(\nabla_{\theta} h\left(\mu+B \epsilon_{1}+c \circ \epsilon_{2}\right) \epsilon_{2}^{\top}+\left(B B^{\top}+D^{2}\right)^{-1}\left(B \epsilon_{1}+c \circ \epsilon_{2}\right) \epsilon_{2}^{\top}\right)\right),
$$

where we have written $\operatorname{diag}(A)$ for the diagonal elements of a square matrix $A$. Here, the inverse matrix $\left(B B^{\top}+D^{2}\right)^{-1}$ can be computed efficiently; see 20 . We note that in the expression for the gradient of $B$ above, we should set to zero the upper 
triangular components which correspond to elements of $B$ which are fixed at zero. Unbiased estimation of gradients for stochastic gradient ascent can proceed based on these expressions by drawing one or more samples from $p_{\epsilon}(\cdot)$ to estimate the expectations.

\subsection{Efficient natural gradient VAFC method}

We now describe how to efficiently compute the natural gradient (15) by leveraging the factor structure (16). Ong et al. (2017b) also considered a natural gradient method for Gaussian variational approximation with factor covariance. However, this was in the context of likelihood-free inference methods where the dimension of $\theta$ is low compared to the models of interest here, and they simply used naive methods for solving the linear systems involving $I_{F}(\lambda)$ required to compute the natural gradient. This is impractical in high-dimensional problems, and here we demonstrate how to implement natural gradient VAFC when $\theta$ is high-dimensional using conjugate gradient methods (see, for example, Stoer (1983)).

Write $I_{F}(\lambda)$ in partitioned form as

$$
I_{F}(\lambda)=\left[\begin{array}{ccc}
I_{11} & I_{21}^{\top} & I_{31}^{\top} \\
I_{21} & I_{22} & I_{32}^{\top} \\
I_{31} & I_{32} & I_{33}
\end{array}\right],
$$

where the blocks in the partition follow the partition of $\lambda$ as $\lambda=\left(\mu^{\top}, \operatorname{vec}(B)^{\top}, c^{\top}\right)^{\top}$. Because the upper triangle of $B$ is fixed at zero the corresponding rows and columns of $I_{F}(\lambda)$ should be omitted. Ong et al. (2017b) show that $I_{11}=\Sigma^{-1}, I_{21}=I_{31}=0, I_{22}=$ $2\left(B^{\top} \Sigma^{-1} B \otimes \Sigma^{-1}\right)$ (where $\otimes$ denotes the Kronecker product), $I_{33}=2\left(D \Sigma^{-1}\right) \circ\left(\Sigma^{-1} D\right)$ and $I_{32}=2\left(B^{\top} \Sigma^{-1} D \otimes \Sigma^{-1}\right) E_{d}^{\top}$, where $E_{d}$ is the $d \times d^{2}$ matrix that picks out the diagonal elements of the $d \times d$ matrix $A$ from its vectorization, so that $E_{d} \operatorname{vec}(A)=$ $\operatorname{diag}(A)$. To use a conjugate gradient linear solver to compute $I_{F}(\lambda)^{-1} \nabla_{\lambda} \operatorname{LB}(\lambda)$ we simply need to be able to compute matrix vector products of the form $I_{F}(\lambda) x$ for any vector $x$ quickly without needing to store the elements of $I_{F}(\lambda)$.

This can be done provided we can do matrix vector products for the matrices $I_{11}, I_{22}, I_{33}$ and $I_{32}$. Except for the one-factor case described below, this is still difficult so we further approximate $I_{F}(\lambda)$ by setting $I_{32}=0$ and replacing $I_{33}$ with $\tilde{I}_{33}=2\left(D \tilde{\Sigma}^{-1}\right) \circ\left(\tilde{\Sigma}^{-1} D\right)$, where $\tilde{\Sigma}$ is the diagonal approximation to $\Sigma$ obtained by setting its off-diagonal elements to zero. Using this approximation and $I_{32}=0$ we obtain a positive definite approximation $\tilde{I}_{F}(\lambda)$ to $I_{F}(\lambda)$ which we use instead of $I_{F}(\lambda)$ in the natural gradient. We note that these approximations do not affect the factor structure of $\Sigma$ in $(16)$.

Multiplications involving $\tilde{I}_{33}$ are simple since this matrix is diagonal, but we still need efficient methods to compute matrix vector products for $I_{11}$ and $I_{22}$. Considering $I_{11}$ first, we note that by using the Woodbury formula

$$
I_{11}=\Sigma^{-1}=D^{-2}-D^{-2} B\left(I+B^{\top} D^{-1} B\right)^{-1} B^{\top} D^{-2},
$$


and then noting that $D$ is diagonal and $\left(I+B^{\top} D^{-2} B\right)$ is $f \times f, f \ll d$, we can calculate $I_{11} x=\Sigma^{-1} x$ without needing to store any $d \times d$ matrix or do any dense $d \times d$ matrix multiplications. Next, consider $I_{22} x$ for some vector $x$. We note that

$$
I_{22}=2\left(B^{\top} \Sigma^{-1} B \otimes \Sigma^{-1}\right) x=2 \operatorname{vec}\left(\Sigma^{-1} x^{*} B^{\top} \Sigma^{-1} B\right),
$$

where $x^{*}$ denotes the $d \times f$ matrix such that $x=\operatorname{vec}\left(x^{*}\right)$ and where we have used the identity that for conformable matrices $X, Y, Z, \operatorname{vec}(X Y Z)=\left(Z^{\top} \otimes X\right) \operatorname{vec}(Y)$. Then $\Sigma^{-1} x^{*}$ is computed efficiently by the Woodbury formula, and similarly for $B^{\top} \Sigma^{-1} B$. We refer to our natural gradient estimation method with $f$ factors as NAGVAC- $f$.

We now consider the special case of the NAGVAC-1 method where the covariance matrix $\Sigma$ is parameterized as in (16) with $B$ a vector. In this case, the natural gradient (15) can be computed efficiently and the computational complexity is $O(d)$. See Algorithm 2, whose detailed derivation is in the Appendix. This estimation method is computationally attractive, especially when the dimension $d$ is extremely large. The experimental studies in Section 6] suggest that in some applications this method is able to produce a prediction accuracy comparable to the accuracy obtained by methods that use more flexible factor decomposition structures of $\Sigma$. Trippe and Turner (2018) discuss the phenomenon where richer variational families produce inferior performance in terms of predictive loss for neural networks models, and provide some theoretical insights into this phenomenon.

Algorithm 2 (Computing the natural gradient). Input: Vector B, c and ordinary gradient vector $g=\left(g_{1}^{\top}, g_{2}^{\top}, g_{3}^{\top}\right)^{\top}$ with $g_{1}$ the vector formed by the first d elements of $g, g_{2}$ formed by the next d elements, and $g_{3}$ the last d elements. Output: The natural gradient $g^{\text {nat }}=I_{F}^{-1} g$.

- Compute the vectors $v_{1}=c^{2}-2 B^{2} \circ c^{-4}, v_{2}=B^{2} \circ c^{-3}$, and the scalars $\kappa_{1}=$ $\sum_{i=1}^{d} b_{i}^{2} / c_{i}^{2}, \kappa_{2}=\frac{1}{2}\left(1+\sum_{i=1}^{d} v_{2 i}^{2} / v_{1 i}\right)^{-1}$.

- Compute

$$
g^{n a t}=\left(\begin{array}{c}
\left(g_{1}^{\top} B\right) B+c^{2} \circ g_{1} \\
\frac{1+\kappa_{1}}{2 \kappa_{1}}\left(\left(g_{2}^{\top} B\right) B+c^{2} \circ g_{2}\right) \\
\frac{1}{2} v_{1}^{-1} \circ g_{3}+\kappa_{2}\left[\left(v_{1}^{-1} \circ v_{2}\right)^{\top} g_{3}\right]\left(v_{1}^{-1} \circ v_{2}\right)
\end{array}\right) \text {. }
$$

\section{Variable selection and regularization priors}

This section first presents a method for variable selection in DeepGLM and DeepGLMM. The method is based on the Bayesian adaptive group Lasso method which is developed for GLM in Leng et al. (2014) and normal regression in Kyung et al. (2010). Consider a neural network as in Figure 1. Denote by $w_{X_{j}}$ the vector of weights that

connect the covariate $X_{j}$ to the $m$, say, units in the first hidden layer. We use the 
following priors

$$
w_{X_{j}}\left|\tau_{j} \sim \mathcal{N}\left(0, \tau_{j} I_{m}\right), \quad \tau_{j}\right| \gamma_{j} \sim \operatorname{Gamma}\left(\frac{m+1}{2}, \frac{\gamma_{j}^{2}}{2}\right), \quad j=1, \ldots, p,
$$

with the $\gamma_{j}>0$ the shrinkage parameters. By the normal-Gamma mixture Andrews and Mallows, 1974; Kyung et al., 2010), we have that

$$
p\left(w_{X_{j}} \mid \gamma_{j}\right)=\int p\left(w_{X_{j}} \mid \tau_{j}\right) p\left(\tau_{j} \mid \gamma_{j}\right) d \tau_{j} \propto \exp \left(-\gamma_{j}\left\|w_{X_{j}}\right\|_{2}\right)
$$

with $\left\|w_{X_{j}}\right\|_{2}$ the $l_{2}$-norm of $w_{X_{j}}$. Hence, the posterior mode of the $w_{X_{j}}$ induced from the above hierarchical prior with the same $\gamma_{j}=\gamma$ is equivalent to the group Lasso estimator of Yuan and Lin (2006).

Selecting the adaptive shrinkage parameters $\gamma_{j}$ is challenging. We develop an empirical Bayes method for estimating these tuning parameters based on an iterative scheme within the variational approximation procedure. Writing the Bayesian hierarchical model in the generic form

$$
y|\psi, \theta \sim p(y \mid \theta, \psi), \quad \theta| \psi \sim p(\theta \mid \psi),
$$

with $y$ the data, $\theta$ the model parameters and $\psi$ the hyperparameters to be selected. The marginal likelihood for $\psi$ is $p(y \mid \psi)=\int p(\theta \mid \psi) p(y \mid \theta, \psi) d \theta$, which can be maximized using an EM-type algorithm (Casella, 2001). Given an initial value $\psi^{(0)}$, we iteratively update $\psi$ by

$$
\psi^{(k+1)}=\operatorname{argmax}_{\psi}\left\{\mathbb{E}_{\theta \mid y, \psi^{(k)}} \log p(y, \theta \mid \psi)\right\},
$$

where $\mathbb{E}_{\theta \mid y, \psi^{(k)}}(\cdot)$ is the expectation with respect to the posterior distribution $p\left(\theta \mid y, \psi^{(k)}\right)$. It can be shown that the updating rule for $\gamma_{j}$ is

$$
\gamma_{j}^{(k+1)}=\sqrt{\frac{m+1}{\mathbb{E}_{\theta \mid y, \gamma_{j}^{(k)}}\left[\tau_{j}\right]}} .
$$

In our variational approximation framework, the expectation $\mathbb{E}_{\theta \mid y, \psi^{(k)}}(\cdot)$ can be naturally approximated by the expectation w.r.t. the current variational approximation $q_{\lambda^{(k)}}(\theta)$, and the updates in 21) can be computed in closed form. One can merge the auxiliary parameters $\tau_{1}, \ldots, \tau_{p}$ into the model parameters and learn them jointly by the Gaussian variational posterior $q_{\lambda}(\theta)$. Alternatively, one can conveniently update the variational posterior for each $\tau_{j}$ separately in a fixed-form within mean-field variational approximation procedure; see, e.g. Tran et al. (2016b). We use the latter in this paper. The optimal VB posterior for $1 / \tau_{j}$ is inverse-Gaussian with mean and shape parameter

$$
\alpha_{\tau_{j}} \leftarrow \frac{\gamma_{j}}{\sqrt{\mathbb{E}_{q_{\lambda}}\left[w_{X_{j}}^{\prime} w_{X_{j}}\right]}}, \quad \beta_{\tau_{j}} \leftarrow \gamma_{j}^{2}
$$


Because $\mathbb{E}_{\theta \mid y, \gamma_{j}^{(k)}}\left[\tau_{j}\right]$ can be approximated by $\mathbb{E}_{q_{\lambda}}\left[\tau_{j}\right]=1 / \alpha_{\tau_{j}}+1 / \beta_{\tau_{j}}$, the update in 21] becomes

$$
\gamma_{j} \leftarrow \sqrt{\frac{m+1}{1 / \alpha_{\tau_{j}}+1 / \beta_{\tau_{j}}}} .
$$

The updates in 22 and (23) are then embedded in the main variational iterate procedure Algorithm 1. This leads to a convenient and principled way for selecting the shrinkage parameters $\gamma_{j}$, which would be very challenging for alternative methods.

In order to control overfitting, we use a ridge-type regularization for the rest of the weights in the neural network. Let $\widetilde{w}$ be the vector of all the weights except those that connect the input layer to the first hidden layer and the intercepts (also called bias terms). We use the following prior for $\widetilde{w}$

$$
p(\widetilde{w}) \propto \exp \left(-\frac{\gamma_{w}}{2} \widetilde{w}^{\top} \widetilde{w}\right)
$$

with $\gamma_{w}$ the shrinkage parameter. Similarly to above, $\gamma_{w}$ is selected by maximizing the marginal likelihood and the update rule is

$$
\gamma_{w} \leftarrow \frac{d_{\widetilde{w}}}{\mathbb{E}_{q_{\lambda}}\left[\widetilde{w}^{\top} \widetilde{w}\right]},
$$

where $d_{\widetilde{w}}$ denotes the dimension of $\widetilde{w}$.

\section{$5 \quad$ Practical recommendations in training DeepGLM and DeepGLMM}

The NAGVAC estimation method can be used as a general estimation method for any model. However, this section focuses on estimating DFNN-based models, and discusses some implementation recommendations that we found useful in practice.

\subsection{Stopping rule and lower bound for model choice}

It is common in deep learning applications of neural network methods to implement early stopping in the optimization to avoid overfitting, because often the training loss decreases steadily over the optimization updates, but the validation loss starts increasing at some point. In our VB framework, the lower bound (12) can be monitored to check the convergence and decide when to stop training. There are two advantages to using the lower bound. The first advantage is that a validation set is unnecessary, which leaves more information for the training phrase. Also, a stopping rule based on a validation set might depend on how the validation set is selected. The second advantage is that, given that appropriate regularization priors on the weights as the ones in Section 4 have been used to control overfitting, the maximized lower 
bound can be used for model choice; see the examples in Section 6. The lower bound (12) can be estimated efficiently using the same sample of $\theta$ generated for computing the gradient vector. To reduce the noise level in estimating the lower bound, we follow Tran et al. (2017) and take the average of the lower bound over a moving window of $K$ iterations. We stop training if this moving averaged lower bound does not improve after $P$ iterations.

\subsection{Learning rate and the momentum method}

We employ the following fixed learning rate which is widely used in the deep learning literature

$$
a_{t}=\epsilon_{0} \frac{\tau}{t}
$$

where $\epsilon_{0}$ is a small value, e.g. 0.01 and $\tau$ is some threshold from which the learning rate is reduced, e.g. $\tau=1000$. It might also be useful to use some adaptive learning rate methods, and later we consider one method adapted from Ranganath et al. (2013) which is described in Ong et al. (2018). This learning rate is suitable for use with the natural gradient.

As a method of accelerating the stochastic gradient optimization we also consider using the momentum method (Polyak, 1964). The update rule is

$$
\begin{aligned}
\overline{\nabla_{\lambda} \mathrm{LB}} & =\alpha_{\mathrm{m}} \overline{\nabla_{\lambda} \mathrm{LB}}+\left(1-\alpha_{\mathrm{m}}\right) \widehat{\nabla_{\lambda} \mathrm{KL}}\left(\lambda^{(t)}\right)^{\mathrm{nat}}, \\
\lambda^{(t+1)} & =\lambda^{(t)}+a_{t} \overline{\nabla_{\lambda} \mathrm{LB}}
\end{aligned}
$$

where $\alpha_{\mathrm{m}} \in[0,1]$ is the momentum weight. The use of the moving average gradient $\overline{\nabla_{\lambda} \mathrm{LB}}$ helps remove some of the noise inherent in the estimated gradients of the lower bound. See Goodfellow et al. (2016), Chapter 8, for a detailed discussion on the usefulness of the momentum method.

\subsection{Activation function and initialization}

For initialization of the variational parameters $\lambda^{(0)}=\left(\mu^{(0)}, B^{(0)}, c^{(0)}\right)$, we follow Glorot and Bengio (2010) and initialize each weight in $\mu^{(0)}$ by the uniform distribution $\mathcal{U}\left(-\sqrt{\frac{6}{m+n}}, \sqrt{\frac{6}{m+n}}\right)$, where the weight connects a layer with $m$ units to a layer with $n$ units. The elements in $B^{(0)}$ are initialized by $\mathcal{N}\left(0,0.01^{2}\right)$ and the elements in $c^{(0)}$ are initialized by 0.01. It is advisable to first standardize the input data so that each column has a zero sample mean and a standard deviation of one. Finally, we use the rectified activation function $h(x)=\max (0, x)$ in all examples, unless otherwise stated. This activation function has a strong connection with biological neuroscience and has been widely used in the deep learning literature; see, e.g., Goodfellow et al. (2016). 


\section{Experimental studies and applications}

To illustrate the performance of variable selection, the prediction accuracy of DeepGLM and DeepGLMM, and the efficiency of the NAGVAC training algorithm, we consider a range of experimental studies and applications. All the examples are implemented in Matlab and run on a desktop computer with i5 3.3 Ghz Intel Quad Core. All the DeepGLM and DeepGLMM models are trained using the NAGVAC-1 method, unless otherwise stated.

We use two predictive measurements. The first is the partial predictive score (PPS)

$$
P P S=-\frac{1}{n_{\text {test }}} \sum_{\left(x_{i}, y_{i}\right) \in \text { test data }} \log p\left(y_{i} \mid x_{i}, \widehat{\theta}\right),
$$

with $\widehat{\theta}$ a point estimate of the model parameters. The second is the mean squared error (MSE),

$$
M S E=\frac{1}{n_{\text {test }}} \sum_{\left(x_{i}, y_{i}\right) \in \text { test data }}\left(y_{i}-\widehat{y}_{i}\right)^{2},
$$

with $\widehat{y}_{i}$ a prediction of $y_{i}$, which is called the misclassification rate (MCR) for binary response $y_{i}$.

\subsection{Experimental studies}

\subsubsection{Efficiency of the NAGVAC algorithm}

We first study the efficiency of the NAGVAC algorithm as a genenal training method. We use the German credit dataset from the UCI Machine Learning Repository, http://archive.ics.uci.edu/ml, with 1000 observations, of which 750 are used as the training data and the rest as the test data. The data consist of a binary response variable, credit status, which is good credit (1) or bad credit (0), together with 30 covariate variables such as education, credit amount, employment status, etc. We consider a simple logistic regression model for predicting the credit status, based on the covariates. We use an improper flat prior for the coefficients $\theta$, i.e. $p(\theta) \propto 1$.

We study the performance of the natural gradient method compared to the ordinary gradient method. Figure 2 shows the convergence of the lower bound of the ordinary gradient method and the NAGVAC-1 method. For the ordinary gradient, we use the adaptive learning rate method ADADELTA of Zeiler (2012). For the natural gradient, we use both the fixed learning rate in (25) and the adaptive learning rate of Ong et al. (2018) (which is based on the method in Ranganath et al. (2013)). The figure shows that both the natural gradient method speed up the convergence significantly. Although incorporating an adaptive learning rate into NAGVAC helps to speed up the convergence, the improvement is not always significant compared to a fixed learning rate. We used the fixed learning rate (25) in all the examples reported below. 


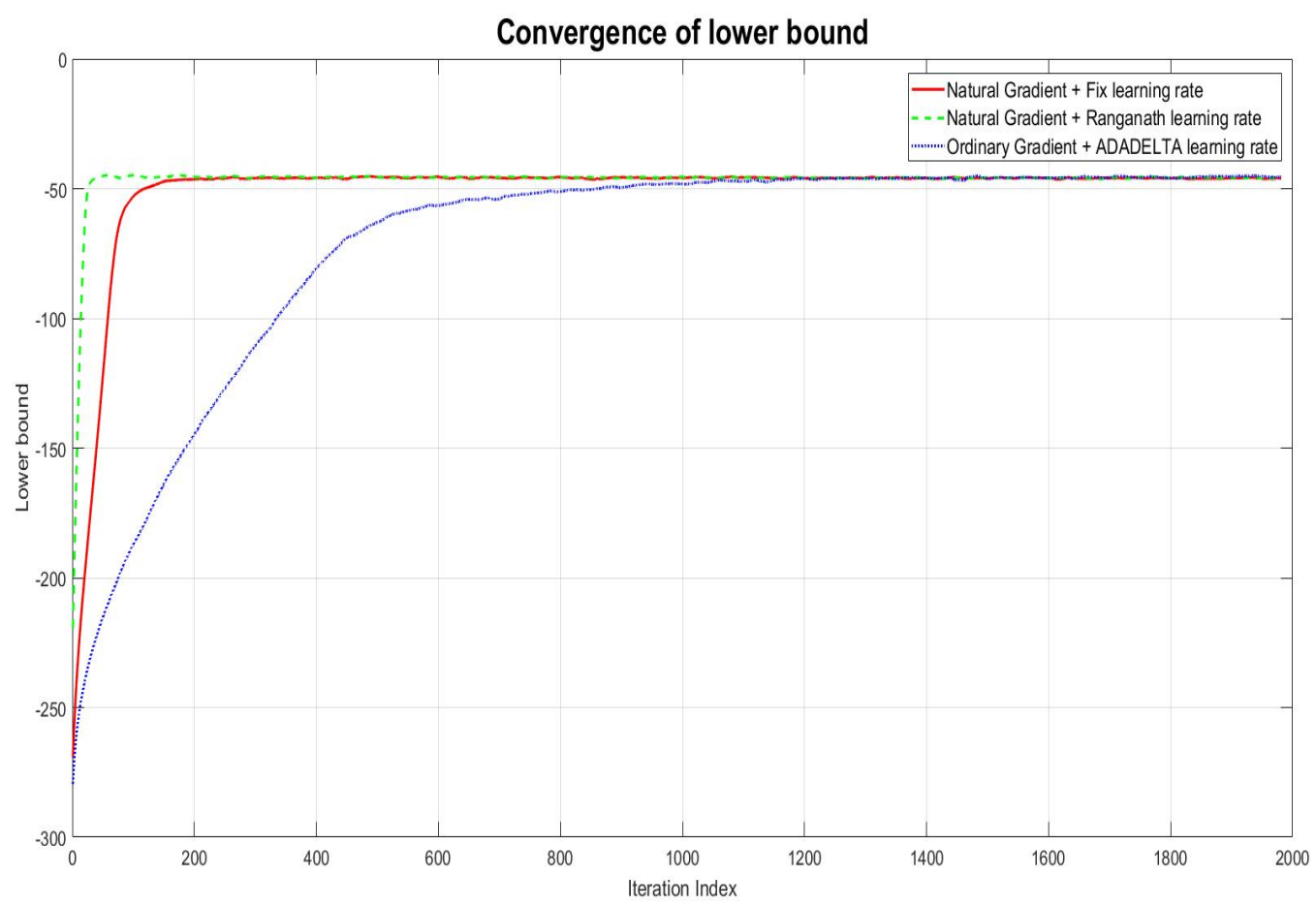

Figure 2: Plot of the lower bound over iterations for the ordinary gradient and NAGVAC-1 methods.

\subsubsection{Variable selection and prediction accuracy of DeepGLM: Binary response}

Data are generated from the following deterministic model

$$
\begin{aligned}
& a=5-2\left(x_{1}+2 x_{2}\right)^{2}+4 x_{3} x_{4}+3 x_{5}+\sum_{i=6}^{20} 0 x_{i}, \\
& y= \begin{cases}1, & a \geq 0, \\
0, & a<0,\end{cases}
\end{aligned}
$$

where the $x_{i}$ are generated from the uniform distribution $\mathcal{U}(-1,1)$. Note that the last 15 variables are irrelevant variables. The training data consist of $n_{\text {train }}=100,000$ observations and the test data of $n_{\text {test }}=100,000$ observations.

We use a neural net with the structure $(20,20,20,1)$, i.e. the input layer has 20 variables, two hidden layers each has 20 units and one scalar output. Figure 3 plots the update of the shrinkage parameters $\gamma_{j}$ as in (23). The shrinkage parameters $\gamma_{6}, \ldots, \gamma_{20}$ w.r.t. the irrelevant variables keep increasing over iterations, while the ones w.r.t. the relevant variables keep decreasing. This shows that the Bayesian adaptive 
group Lasso with the empirical Bayes method for updating the shrinkage parameters is able to scan out correctly irrelevant covariates.

We now compare the predictive performance of DeepGLM to the Bayesian Additive Regression Tree method (BART) of Chipman et al. (2010). BART is a commonly used nonparametric regression method in the statistics literature and is well known for its prediction accuracy and its ability to capture nonlinearity effects. Table 1 summarizes the prediction performance of DeepGLM compared to that of the conventional GLM and BART. GLM, which is logistic regression in this example, does just slightly better than a random guess with a MCR of 40.84\%. As shown, DeepGLM works very well in this example and outperforms both GLM and BART. All the comparisons with BART in this paper are done using the $\mathrm{R}$ package BART (the lattest version 1.6) with default settings for the tuning parameters.

\begin{tabular}{l|cc}
\hline \hline Method & PPS & MCR (\%) \\
\hline GLM & 0.67 & 40.84 \\
BART & 0.08 & 3.09 \\
DeepGLM & 0.03 & 1.03 \\
\hline \hline
\end{tabular}

Table 1: Binary response simulation: Performance of DeepGLM v.s. GLM and BART in term of the partial predictive score (PPS) and the misclassification rate (MCR). Both are evaluated on the test data.

\subsubsection{Variable selection and prediction accuracy of DeepGLM: Continu- ous response}

We generate data from the following highly nonlinear model

$y=5+10 x_{1}+\frac{10}{x_{2}^{2}+1}+5 x_{3} x_{4}+2 x_{4}+5 x_{4}^{2}+5 x_{5}+2 x_{6}+\frac{10}{x_{7}^{2}+1}+5 x_{8} x_{9}+5 x_{9}^{2}+5 x_{10}+\epsilon$,

where $\epsilon \sim \mathcal{N}(0,1),\left(x_{1}, \ldots, x_{20}\right)^{\top}$ are generated from a multivariate normal distribution with mean zero and covariance matrix $\left(0.5^{|i-j|}\right)_{i, j}$. Note that the last 10 variables are irrelevant variables. The training data has 100,000 observations and the test data has 20,000 .

Figure 4 plots the update of the shrinkage parameters $\gamma_{j}$ as in $(23)$. All the shrinkage parameters w.r.t. the irrelevant variables keep increasing over iterations, while the ones w.r.t. the relevant variables, except $\gamma_{8}$, keep decreasing. The reason is that the main effect of $x_{8}$ is not included into model (26), hence the signal of $x_{8}$ might not be strong enough to be identifiable. Table 2 summarizes the prediction performance of DeepGLM in comparison to the conventional GLM and BART. DeepGLM works well in this example and ourperforms both GLM and BART. 


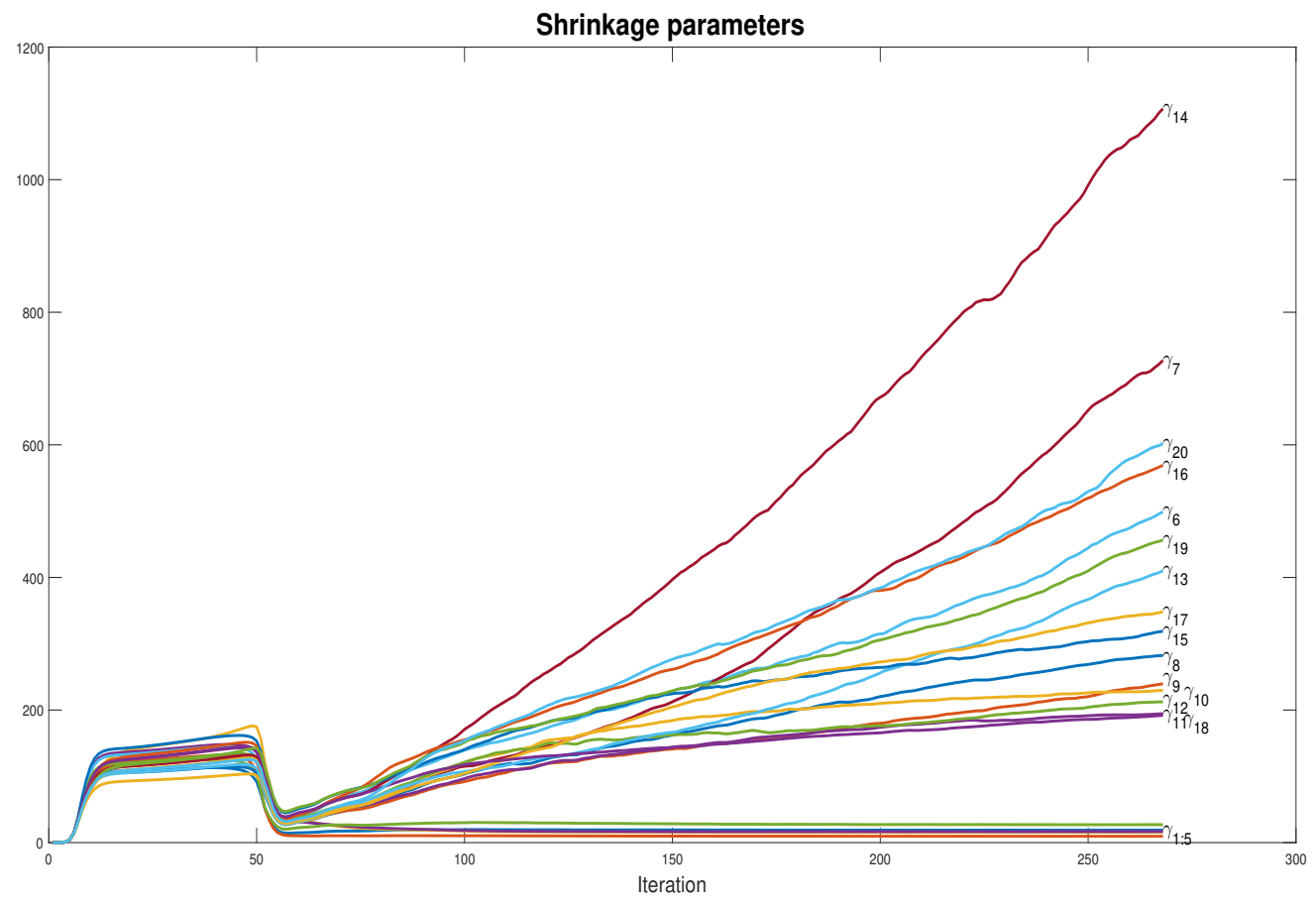

Figure 3: Plots of the shrinkage parameters $\gamma_{j}$ over iterations. The shrinkage parameters w.r.t the irrelevant variables keep increasing, while the ones w.r.t the relevant variables keep decreasing.

\subsubsection{Prediction accuracy of DeepGLMM: binary panel data simulation}

We study the DeepGLMM on a simulation binary panel dataset $D=\left\{\left(x_{i t}, y_{i t}\right) ; t=\right.$ $1, \ldots, 20 ; i=1, \ldots, 1000\}$ with $x_{i t}$ the vector of covariates and $y_{i t}$ the response of subject $i$ at time $t$. The response $y_{i t}$ is generated from the following model:

$$
\begin{aligned}
& a_{i t}=2+3\left(x_{i t, 1}-2 x_{i t, 2}\right)^{2}-5 \frac{x_{i t, 3}}{\left(1+x_{i t, 4}\right)^{2}}-5 x_{i t, 5}+b_{i}+\epsilon_{i t}, \\
& y_{i t}= \begin{cases}1, & \text { if } a_{i t}>0, \\
0, & \text { otherwise }\end{cases}
\end{aligned}
$$

where $b_{i} \sim \mathcal{N}(0,0.1)$ is a random intercept representing charactersistics of subject $i$ and $\epsilon_{i t} \sim \mathcal{N}(0,1)$ is random noise associated with reponses $y_{i t}$. The $x_{i t, j}, j=1, \ldots, 5$, are generated from a uniform distribution $\mathcal{U}(-1,1)$.

We fit the following DeepGLMM to this dataset

$$
y_{i t} \mid x_{i t} \sim \operatorname{Binomial}\left(1, \mu_{i t}\right), \quad \log \left(\frac{\mu_{i t}}{1-\mu_{i t}}\right)=\mathfrak{N}\left(x_{i t}, w, \beta+\alpha_{i}\right),
$$




\begin{tabular}{lcc}
\hline \hline Method & PPS & MSE \\
\hline GLM & 3.31 & 275.5 \\
BART & 1.49 & 4.89 \\
DeepGLM & 1.20 & 4.07 \\
\hline \hline
\end{tabular}

Table 2: Continuous response simulation: Performance of DeepGLM v.s. conventional GLM and BART in term of the partial predictive score (PPS) and the mean squared error (MSE). Both are evaluated on the test data. The structure of the neural net is $(20,20,20,1)$.

where $\mathfrak{N}\left(x_{i t}, w, \beta+\alpha_{i}\right)$ is the scalar output of a neural net with input $x_{i t}$, inner weights $w$ and output weights $\beta+\alpha_{i}$. We assume that the random effects $\alpha_{i} \sim \mathcal{N}(0, \Gamma)$ with $\Gamma=\operatorname{diag}\left(\Gamma_{0}, \ldots, \Gamma_{m}\right)$. The model parameters are $\theta=\left(w, \beta, \Gamma_{0}, \ldots, \Gamma_{m}\right)$. We use Gamma priors on the $\Gamma_{j}, \Gamma_{j} \sim \operatorname{Gamma}\left(a_{0}, b_{0}\right)$, and set the hyperparameters $a_{0}=1$ and $b_{0}=0.1$ in this example. The Appendix gives further details on training this model.

For each subject, we use the first 17 observations for training and the last 3 observations for testing. That is, we are interested in the within-subject prediction. The neural network has one hidden layer with 10 nodes, and we compare the DeepGLMM model with the conventional logistic regression model with a random intercept.

For binary panel data, the misclassification rate is defined as follows. Let $\bar{\theta}$ be the mean of the VB approximation $q_{\lambda}(\theta)$ after convergence. Let $\widehat{\mu}_{\alpha_{i}}$ in $(28)$ be the mode of $p\left(\alpha_{i} \mid y_{i}, x_{i}, \bar{\theta}\right)$, which can be used as the prediction of the random effects $\alpha_{i}$ of subject $i$. Let $\left(y_{i t_{0}}, x_{i t_{0}}\right)$ be a future data pair. We set the prediction of $y_{i t_{0}}$ as

$$
\widehat{y}_{i t_{0}}=1 \text { if and only if } \mathfrak{N}\left(x_{i t_{0}}, w, \beta+\widehat{\mu}_{\alpha_{i}}\right) \geq 0 .
$$

The classification error is

$$
\mathrm{MCR}=\sum I_{\widehat{y}_{i t_{0}} \neq y_{i t_{0}}} / \text { total number of future observations }
$$

with the sum over the test data points $\left(y_{i t_{0}}, x_{i t_{0}}\right)$.

\begin{tabular}{lll}
\hline \hline Model & PPS & MCR \\
\hline GLMM & 1.24 & $17.57 \%$ \\
DeepGLMM & 0.13 & $5.27 \%$ \\
\hline \hline
\end{tabular}

Table 3: Simulation binary panel dataset: Performance of DeepGLMM v.s. GLMM in term of the partial predictive score (PPS) and the misclassification rate (MCR). Both are evaluated on the test data.

Table 3 summarizes the performance of DeepGLMM and GLMM. The results show that modelling covariate effects in a flexible way using the neural network basis functions is helpful here in terms of improving both PPS and MCR. 


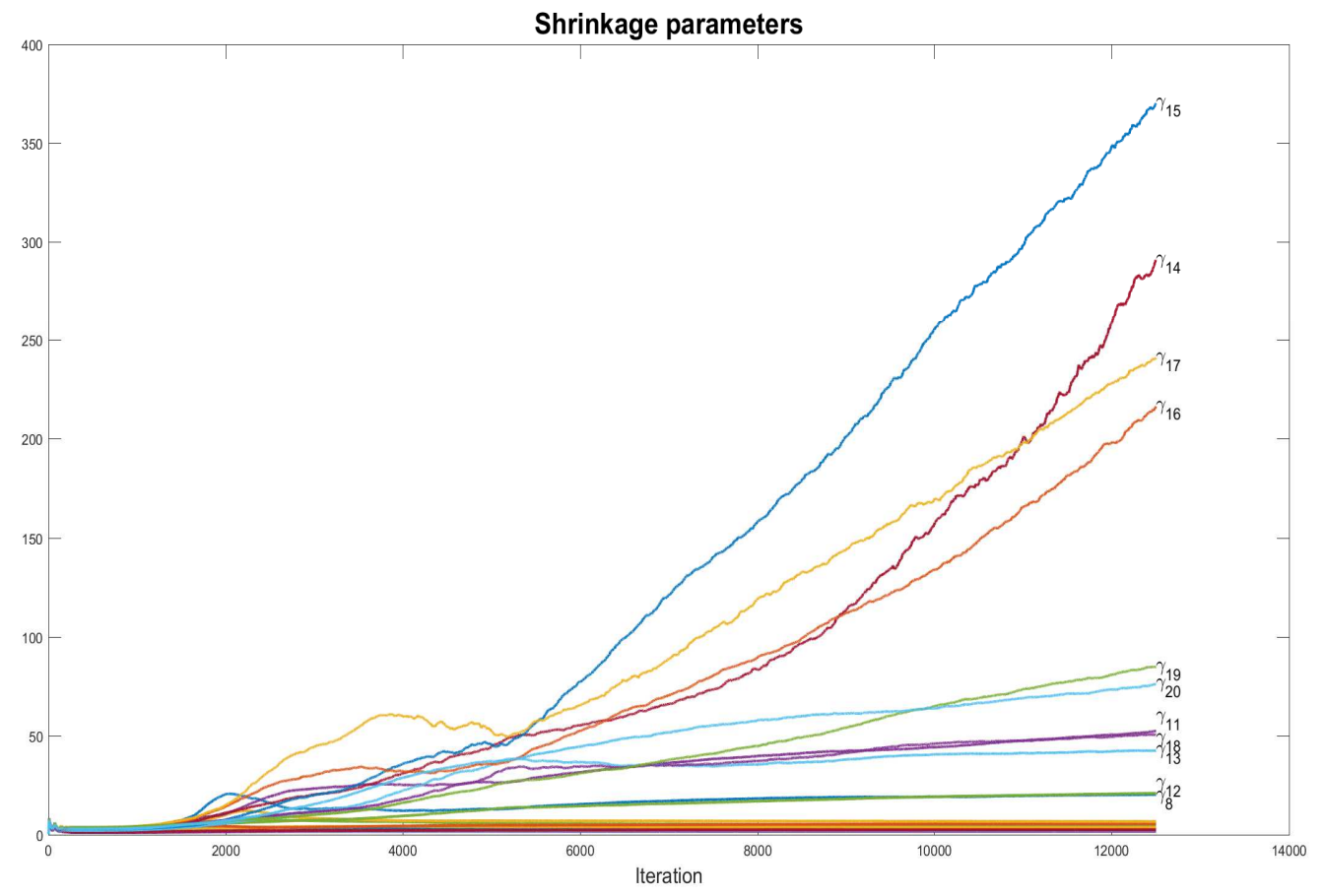

Figure 4: Plots of the shrinkage parameters $\gamma_{j}$ over iterations. The shrinkage parameters w.r.t. the irrelevant variables keep increasing, while the ones w.r.t. the relevant variables keep decreasing.

\subsection{Applications}

\subsubsection{Direct Marketing data}

We consider the Direct Marketing dataset used in the statistics textbook of Jank (2011). This dataset consists of 1000 observations, of which 900 were used for training and the rest for testing. The response is the amount (in \$1000) a customer spends on the company's products per year. There are 11 covariates including gender, income, the number of ads catalogs, married status, young, old, etc. The careful analysis in Jank (2011) shows that the ordinary linear regression model fits well to this dataset. We first wish to explore the significance of the covariates in terms of explaining the response $y$. We tried many DeepGLM models with one hidden layer neural network and the number of units varying, the plots of the shrinkage parameters over iterations have a consistent pattern and all show that the shrinkage parameters with respect to the covariates married, gender, home owner, old and young increase over iterations. The left panel of Figure 5 plots the shrinkage parameters from a DeepGLM with 6 hidden units, with an MSE of 0.2325. The plot suggests that these five covariates can be removed from the model. The right panel of Figure 5 plots the shrinkage 
parameters from a DeepGLM with a neural net structure $(6,6,6,1)$, after the five insignificant covariates have been removed. The MSE for this model is 0.1718. Table 4 shows that DeepGLM gives a better predictive performance than its competitors. We note, however, that without considering variable selection, we could not successfully train a DeepGLM model that has a better predictive performance than BART.
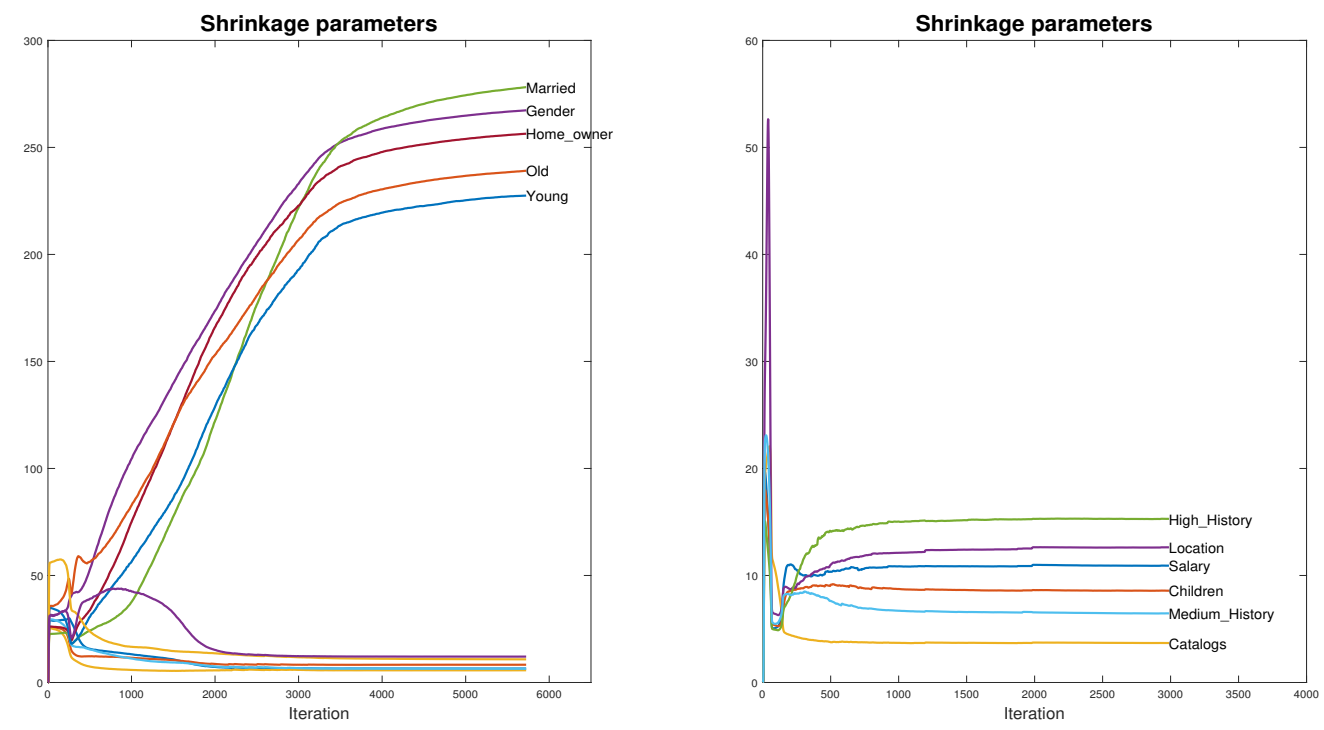

Figure 5: Plots of the shrinkage parameters over iterations. Left panel: The shrinkage parameters from a neural net with 6 hidden units. Right panel: The shrinkage parameters from a $(6,6,6,1)$ neural net, after the five insignificant covariates have been removed.

\begin{tabular}{lll}
\hline \hline Model & PPS & MSE \\
\hline GLM & -0.11 & 0.29 \\
BART & -0.35 & 0.18 \\
DeepGLM & -0.38 & 0.17 \\
\hline \hline
\end{tabular}

Table 4: Direct marketing data after the five insignificant covariates have been removed: Performance of GLM, BART and DeepGLM. Neural net structure is $(6,6,6,1)$.

\subsubsection{Abalone data}

The abalone data, available on the UCI Machine Learning Repository, is a benchmark dataset that has been used in many regression analysis papers. The data has 4177 observations of which $85 \%$ were used for training and the rest for testing. We first 
explore the use of the lower bound for model selection. Table 5 summarizes the lower bound and MSE (computed on the test data), averaged over 10 different runs, for various neural network structures. Here, we use the MSE on the test data in order to assess the usefulness of the lower bound as a model selection criterion. The results suggest that, in general, a small lower bound leads to a worse MSE and that a DeepGLM with either neural net $(9,5,5,1)$ or $(9,10,10,1)$ can be selected. We conducted the same experiment for other structures $(9,6,6,1),(9,7,7,1),(9,8,8,1)$ and $(9,9,9,1)$ and observed a little change in both LB and MSE. This result is consistent with the findings that have been long realized in the deep learning literature (Bengio, 2012 ) that a small change around a neural net structure that works well does not affect the predictive performance appreciably. In Table 5, the neural nets $(9,5,5,1)$ and $(9,10,10,1)$ have similar LB, but the former should be selected as it has a simpler structure. This experimental exploration illustrates the attractiveness of using the lower bound as a model selection tool in our NAGVAC method.

\begin{tabular}{l|cccc}
\hline \hline Structure & {$[9,2,2,1]$} & {$[9,5,5,1]$} & {$[9,10,10,1]$} & {$[9,20,20,1]$} \\
\hline LB & $-2.212(0.019)$ & $-2.193(0.012)$ & $-2.190(0.010)$ & $-2.446(0.060)$ \\
MSE & $5.17(0.32)$ & $4.71(0.12)$ & $4.74(0.13)$ & $8.61(1.52)$ \\
\hline \hline
\end{tabular}

Table 5: Abalone data: Lower bound LB and MSE (on the test data), averaged over 10 runs, for various neural network structures. The numbers in brackets are standard errors.

One of the attractive features of DeepGLM is that, as a Bayesian method, it offers an easy and principled way to construct the prediction intervals for test data. The predictive distribution of the response $y$ given covariate vector $x$ and data $D$ is

$$
p(y \mid x, D)=\int p(y \mid x, \theta) p(\theta \mid D) d \theta \text {. }
$$

As we approximate the posterior $p(\theta \mid D)$ by the VB distribution $q_{\lambda}(\theta)$ which we can sample from, it is possible to sample from $p(y \mid x, D)$ (assuming that it is easy to sample from $p(y \mid x, \theta)$, which is the case all of our applications). Based on this sample from the predictive distribution, we can compute prediction intervals for the mean $\mathbb{E}(y \mid x, D)$. Figure 6 shows the one standard deviation prediction intervals for the test data.

\subsubsection{Census income data}

This census dataset was extracted from the U.S. Census Bureau database and is available on the UCI Machine Learning Repository. The prediction task is to determine whether a person's income is over $\$ 50 \mathrm{~K}$ per year, based on 14 attributes including age, workclass, race, etc, of which many are categorical variables. After using dummy variables to represent the categorical variables, there are 103 input variables. There 


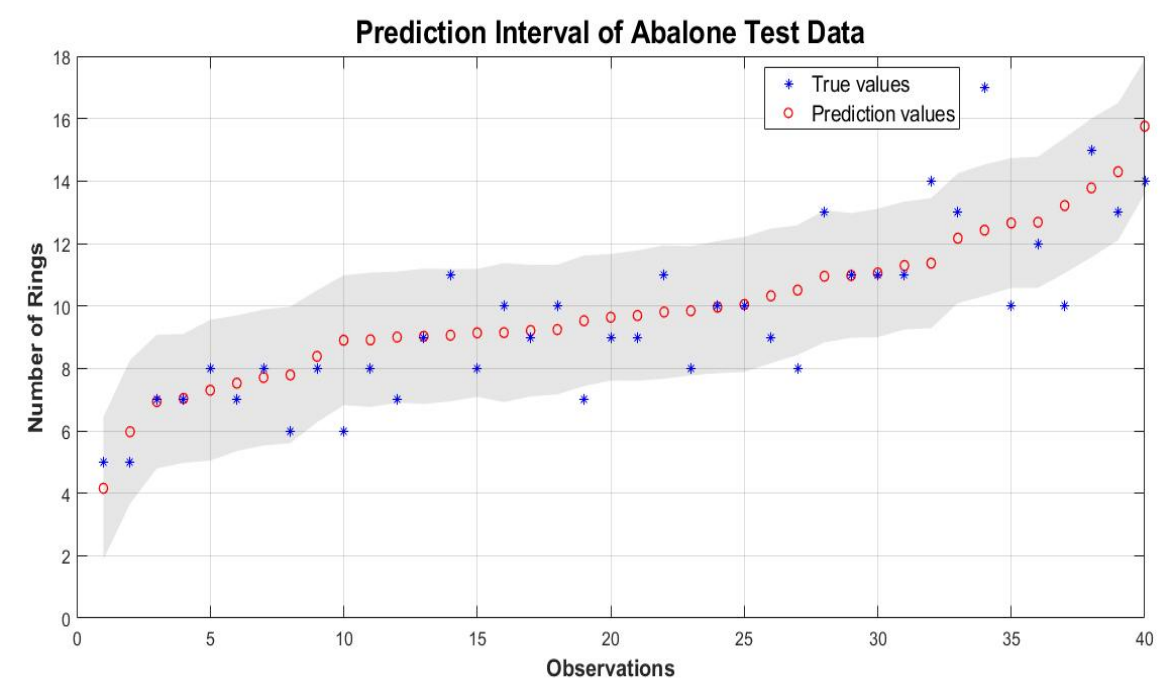

Figure 6: Abalone data: Point prediction and one standard deviation prediction intervals (the shaded area) calculated from the predictive distribution in (27).

\begin{tabular}{lll}
\hline \hline Model & PPS & MSE \\
\hline BART & $1.30(0.01)$ & $4.88(0.06)$ \\
DeepGLM & $1.28(0.01)$ & $4.71(0.12)$ \\
\hline \hline
\end{tabular}

Table 6: Abalone data: Performance of BART and DeepGLM. The neural net structure is $(9,5,5,1)$. The values are averaged over 10 runs with the standard errors in brackets.

are 45221 observations without missing data, of which $33.3 \%$ are kept for testing, the rest are used for training. Table 7 summarizes the predictive performance and Figure 7 plots the ROC curves of DeepGLM and BART, which show that DeepGLM gives slightly better prediction accuracy than BART. Both DeepGLM and BART are run once with a fixed random seed.

\subsubsection{A continuous panel data set: Cornwell and Rupert data}

This section analyzes a continuous panel data set originally analyzed in Cornwell and Rupert (1988). This is a balanced panel dataset with 595 individuals and 4165 observations, each individual was observed for 7 years. The dataset is available from the website of the textbook Baltagi (2013). The variables are listed in Table 8.

We are interested in predicting the wage (on the log scale) of each individual, given the covariates. Let $y_{i t}$ be a continuous variable indicating the log of wage of person $i$ with the vector of covariates $x_{i t}$ in year $t, t=1, \ldots, 7$. We use the following 


\begin{tabular}{lll}
\hline \hline Model & PPS & MCR (\%) \\
\hline BART & 0.68 & 18.6 \\
DeepGLM & 0.34 & 16.09 \\
\hline \hline
\end{tabular}

Table 7: Census data: Performance of DeepGLM v.s. BART. We use a one hidden layer neural net with 40 units.

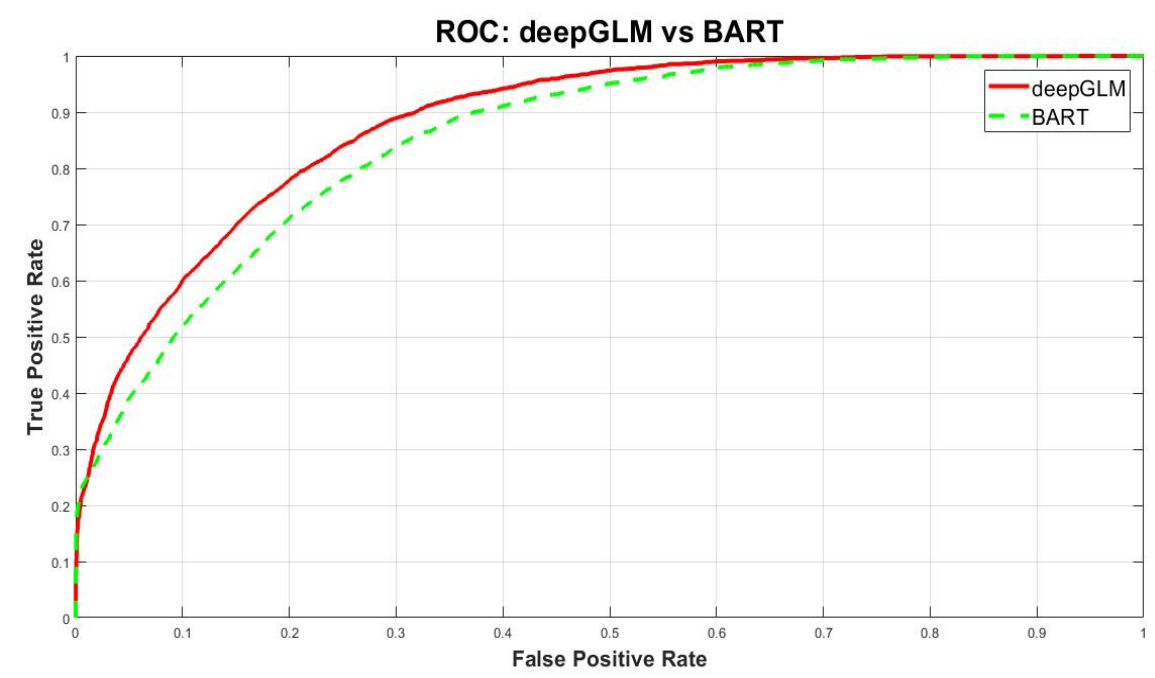

Figure 7: Census data: The ROC curves of DeepGLM v.s. BART. The area under the curve of DeepGLM is larger than that of BART, which suggests that DeepGLM has a better predictive performance in this example.

DeepGLMM model

$$
y_{i t} \mid x_{i t} \sim \mathcal{N}\left(\mu_{i t}, \sigma^{2}\right), \quad \mu_{i t}=\mathfrak{N}\left(x_{i t}, w, \beta+\alpha_{i}\right),
$$

where $\mathfrak{N}\left(x_{i t}, w, \beta+\alpha_{i}\right), \beta$ and $\alpha_{i}$ have the same interpretation as in Section 6.1.4, and $\sigma^{2}$ is the noise variance.

Since we are interested in within-subject prediction, for each individual, we use the first 5 observations as training data, and the last 2 observations for test data. We use a neural network with 2 hidden layers with 5 nodes each; this structure was selected after some experiments using the lower bound as the model selection criterion. We compare the performance of DeepGLMM to a linear regression model with a random effect, using PPS and MSE as evaluation metrics. Table 9 summarizes the results, which show that using the neural network basis functions to model covariate effects in a flexible way can significantly improve both PPS and MSE. 


\begin{tabular}{ll}
\hline \hline Variable & Meaning \\
\hline EXP & Years of full-time work experience \\
WKS & Weeks worked \\
OCC & blue-collar occupation $=1 ;$ otherwise $=0$ \\
IND & manufacturing industry $=1$; otherwise $=0$ \\
SOUTH & south residence $=1 ;$ otherwise $=0$ \\
SMSA & metropolitan residence $=1$, otherwise $=0$ \\
MS & married $=1 ;$ otherwise $=0$ \\
FEM & female $=1 ;$ otherwise $=0$ \\
UNION & Union contract wage $=1 ;$ otherwise $=0$ \\
ED & Years of education \\
BLK & black $=1 ;$ otherwise $=0$ \\
LWAGE & log of wage \\
\hline \hline
\end{tabular}

Table 8: Cornwell and Rupert data: variables and their meaning

\begin{tabular}{lll}
\hline \hline Model & PPS & MSE \\
\hline GLMM & 0.05 & 0.18 \\
DeepGLMM & -0.87 & 0.05 \\
\hline \hline
\end{tabular}

Table 9: Cornwell and Rupert data: Performance of DeepGLMM v.s. conventional GLMM in term of the partial predictive score (PPS) and the mean square error (MSE). Both are evaluated on the test dataset.

\subsubsection{Cancer data: high-dimensional logistic regression using the horse- shoe prior}

This section illustrates that the training method NAGVAC can be used as a general estimation method for high-dimensional models rather than neural network based models. The application is concerned with high-dimensional logistic regression using a sparse signal shrinkage prior, the horseshoe prior (Carvalho et al., 2010). Here the variational optimization is challenging because of the strong dependence between local variance parameters and the corresponding coefficients. Using three real datasets we show that the natural gradient estimation method improves the performance of the approach described in Ong et al. (2017a).

Let $y_{i} \in\{0,1\}$ be a binary response with the corresponding covariates $x_{i}=\left(x_{i 1}, \ldots, x_{i p}\right)^{\top}$, $i=1, \ldots, n$. We consider the logistic regression model

$$
\log \frac{\mu_{i}}{1-\mu_{i}}=\beta_{0}+x_{i}^{\top} \beta,
$$

where $\mu_{i}=\mathrm{P}\left(y_{i}=1 \mid x_{i}\right), \beta_{0}$ is an intercept and $\beta=\left(\beta_{1}, \ldots, \beta_{p}\right)^{\top}$ are coefficients. We 
consider the setting where $p$ is large, possibly $p \gg n$, and use the horseshoe prior for $\beta$ (Carvalho et al., 2010). Specifically we assume $\beta_{0} \sim N(0,10)$ and

$$
\beta_{j} \mid \lambda_{j} \sim N\left(0, \lambda_{j}^{2} g^{2}\right), \quad \lambda_{j} \sim C^{+}(0,1), j=1, \ldots, p, \quad g \sim C^{+}(0,1),
$$

where $C^{+}(0,1)$ denotes the half-Cauchy distribution. The parameters $\lambda_{j}, j=1, \ldots, p$, are local variance parameters providing shrinkage for individual coefficients, and the parameter $g$ is a global shrinkage parameter which can adapt to the overall level of sparsity. The above prior settings are the same as those considered in Ong et al. (2017a).

The parameter $\theta$ consists of $\theta=\left(\beta_{0}, \beta^{\top}, \delta^{\top}, \gamma\right)^{\top}$, where $\delta=\left(\delta_{1}, \ldots, \delta_{p}\right)^{\top}=\left(\log \lambda_{1}, \ldots, \log \lambda_{p}\right)^{\top}$, and $\gamma=\log g$. We consider Gaussian variational approximation for the posterior of $\theta$, using a factor covariance structure. The three gene expression datasets are the Colon, Leukaemia and Breast cancer datasets found at http://www.csie.ntu. edu.tw/ cjlin/libsvmtools/datasets/binary.html. These three datasets Colon, Leukaemia and Breast have training sample sizes of 42, 38 and 38 and test set sizes of 20,34 and 4 respectively. The number of covariates is $p=2000$ for the Colon data, and $p=7120$ for the Leukaemia and Breast datasets. This means that for the Leukaemia and Breast datasets the dimension of $\theta$ is 14,242 so these are examples with a high dimensional parameter. These data were also considered in Ong et al. (2017a) where slow convergence in the variational optimization was observed using their method; we show here that a natural gradient approach offers a significant improvement.

\begin{tabular}{|c|ccc|ccc|}
\hline & \multicolumn{2}{|c|}{ VAFC of } & Ong et al. & \multicolumn{4}{c|}{ NAGVAC-4 } & \multicolumn{4}{c|}{ NAG } & & \\
\hline & Train Error & Test Error & CPU & Train Error & Test Error & CPU \\
\hline Colon & $0 / 42$ & $0 / 20$ & 4.92 & $0 / 42$ & $0 / 20$ & 0.17 \\
Leukemia & $0 / 38$ & $6 / 34$ & 61 & $0 / 38$ & $1 / 34$ & 0.56 \\
Breast & $0 / 38$ & $1 / 4$ & 61.6 & $0 / 38$ & $0 / 4$ & 0.56 \\
\hline
\end{tabular}

Table 10: Performance of the ordinary gradient VAFC and natural gradient VAFC methods on three cancer datasets. Training and test errors rates are reported as the ratio of misclassified data points over the number of data points. Computational time CPU (per 100 iterations) is measured in second.

Table 10 compares the performance of VAFC of Ong et al. (2017a) and our NAGVAC training methods. The table shows the predictive performance and computational time on three cancer datasets. We follow Ong et al. (2017a) and run VAFC with $f=4$ factors and use only a single sample to estimate the gradient of lower bound $(S=1)$ in two methods. As shown, the NAGVAC training method significantly improves the performance of VAFC. 


\section{Discussions and conclusions}

This paper is concerned with flexible versions of generalized linear and generalized linear mixed models where DFNN methodology is used to automatically choose transformations of the raw covariates. The challenges of Bayesian computation are addressed using variational approximation methods with a parsimonious factor covariance structure. We have demonstrated that a natural gradient approach to the variational optimization with this family of approximations is feasible even in high dimensions. Our Bayesian treatment offers a principled and convenient way for selecting the tuning parameters, quantifying uncertainty and doing model selection. Using simulated and real datasets and several different models we show that the improvement that these methods can bring in terms of speed of convergence and computation time are substantial, and that the use of neural network basis functions with random effects is a class of models that deserve more consideration in the literature.

\section{Acknowledgments}

Nghia Nguyen and Robert Kohn were partially supported by the Australian Research Council Center of Excellence grant CE140100049.

\section{Appendix}

\section{Rank-1 natural gradient VB estimation method}

Recall that $\Sigma=B B^{\top}+D^{2}$ with $B=\left(b_{1}, \cdots, b_{d}\right)^{\top}$ and $D=\operatorname{diag}(c), c=\left(c_{1}, \cdots, c_{d}\right)^{\top}$. Then,

$$
\Sigma^{-1}=D^{-2}-\frac{1}{1+\kappa} D^{-2} B B^{\top} D^{-2}, \kappa_{1}=B^{\top} D^{-2} B=\sum \frac{b_{i}^{2}}{c_{i}^{2}}
$$

and $B^{\top} \Sigma^{-1} B=\kappa_{1} /\left(1+\kappa_{1}\right)$. Hence, $I_{22}^{-1}=\frac{1+\kappa_{1}}{2 \kappa_{1}} \Sigma$. We still set $I_{23}=0$ but $I_{33}$ can be computed analytically as follows. Let $\Sigma^{-1}=D^{-2}-h h^{\top}$ with $h=D^{-2} B=\left(1 / \sqrt{1+\kappa_{1}}\right) B \circ$ $c^{-2}$ and $h^{2}=h \circ h$.

$$
\begin{aligned}
I_{33} & =2 D\left(\Sigma^{-1} \circ \Sigma^{-1}\right) D \\
& =2\left(D^{-2}-D\left(h h^{\top}\right) \circ D^{-1}-D^{-1} \circ D\left(h h^{\top}\right)+D\left\{\left(h h^{\top}\right) \circ\left(h h^{\top}\right)\right\} D\right) \\
& =2\left(\operatorname{diag}\left(c^{2}-2 h^{2}\right)+D h^{2}\left(D h^{2}\right)^{\top}\right) \\
& =2\left(\operatorname{diag}\left(v_{1}\right)+v_{2} v_{2}^{\top}\right),
\end{aligned}
$$

with $v_{1}=c^{2}-2 h^{2}$ and $v_{2}=D h^{2}=c \circ h^{2}$. Then,

$$
I_{33}^{-1}=\frac{1}{2}\left(\operatorname{diag}\left(v_{1}^{-1}\right)+\frac{1}{1+\sum v_{2 i}^{2} / v_{1 i}}\left(v_{1}^{-1} \circ v_{2}\right)\left(v_{1}^{-1} \circ v_{2}\right)^{\top}\right)
$$


It's important to note that it's unnecessary to store the matrix $I_{F}^{-1}$. To obtain the natural gradient, all we need is the matrix-vector product of the form $I_{F}^{-1} g$. Write $g=\left(g_{1}^{\top}, g_{2}^{\top}, g_{3}^{\top}\right)^{\top}$ with $g_{1}$ the vector formed by the first $d$ elements of $g, g_{2}$ the vector formed by the next $d$ elements, and $g_{3}$ the last $d$ elements in $g$. The natural gradient is

$$
g^{\mathrm{nat}}=\left(\begin{array}{c}
\left(g_{1}^{\top} B\right) B+c^{2} \circ g_{1} \\
\frac{1+\kappa_{1}}{2 \kappa_{1}}\left(\left(g_{2}^{\top} B\right) B+c^{2} \circ g_{2}\right) \\
\frac{1}{2} v_{1}^{-1} \circ g_{3}+\kappa_{2}\left[\left(v_{1}^{-1} \circ v_{2}\right)^{\top} g_{3}\right]\left(v_{1}^{-1} \circ v_{2}\right)
\end{array}\right),
$$

with $\kappa_{2}=\frac{1}{2}\left(1+\sum_{1}^{d} v_{2 i}^{2} / v_{1 i}\right)^{-1}$. The complexity of computing the natural gradient is $O(d)$.

\section{Further details for the example in Section 6.1.4}

The likelihood contribution w.r.t. the panel $\left(y_{i}, x_{i}\right)$ is

$$
\begin{aligned}
L_{i}(\theta) & =\int p\left(y_{i} \mid x_{i}, w, \beta, \alpha_{i}\right) p\left(\alpha_{i} \mid \Gamma\right) d \alpha_{i} \\
& =\int \exp \left(\sum_{t=1}^{T_{i}} y_{i t} \mathfrak{N}\left(x_{i t}, w, \beta+\alpha_{i}\right)-\log \left(1+e^{\mathfrak{N}\left(x_{i t}, w, \beta+\alpha_{i}\right)}\right)\right) p\left(\alpha_{i} \mid \Gamma\right) d \alpha_{i} .
\end{aligned}
$$

By Fisher's identity (Gunawan et al., 2017)

$\nabla_{\theta} \ell_{i}(\theta)=\int \nabla_{\theta}\left\{\log p\left(\alpha_{i} \mid \Gamma\right)+\sum_{t=1}^{T_{i}} y_{i t} \mathfrak{N}\left(x_{i t}, w, \beta+\alpha_{i}\right)-\log \left(1+e^{\mathfrak{N}\left(x_{i t}, w, \beta+\alpha_{i}\right)}\right)\right\} p\left(\alpha_{i} \mid y_{i}, x_{i}, \theta\right) d \alpha_{i}$.

We have,

$$
\begin{aligned}
p\left(\alpha_{i} \mid y_{i}, x_{i}, \theta\right) \propto & p\left(\alpha_{i} \mid \Gamma\right) p\left(y_{i} \mid x_{i}, w, \beta, \alpha_{i}\right) \\
\propto & \exp \left(\sum_{t=1}^{T_{i}}\left[y_{i t} z_{i t}^{\top}\left(\beta+\alpha_{i}\right)-\log \left(1+e^{z_{i t}^{\top}\left(\beta+\alpha_{i}\right)}\right)\right]-\frac{1}{2} \alpha_{i}^{\top} \Gamma^{-1} \alpha_{i}\right)=\exp \left(f\left(\alpha_{i}\right)\right) . \\
& \nabla_{\alpha_{i}} f\left(\alpha_{i}\right)=\boldsymbol{Z}_{i}^{\top}\left(y_{i}-p_{i}\right)-\Gamma^{-1} \alpha_{i}, p_{i}=p_{i}\left(\alpha_{i}\right)=\left(p_{i 1}, \ldots, p_{i T_{i}}\right)^{\top} \\
& \nabla_{\alpha_{i} \alpha_{i}^{\top}} f\left(\alpha_{i}\right)=-\boldsymbol{Z}_{i}^{\top} \operatorname{diag}\left(p_{i} \circ\left(1-p_{i}\right)\right) \boldsymbol{Z}_{i}-\Gamma^{-1} .
\end{aligned}
$$

Let $\widehat{\mu}_{\alpha_{i}}$ be the maximizer of $f\left(\alpha_{i}\right)$ which can be obtained by the Newton-Raphson method, and let

$$
\widehat{\Sigma}_{\alpha_{i}}=\left(-\left.\nabla_{\alpha_{i} \alpha_{i}^{\top}} f\left(\alpha_{i}\right)\right|_{\alpha_{i}=\widehat{\mu}_{\alpha_{i}}}\right)^{-1}=\left(\boldsymbol{Z}_{i}^{\top} \operatorname{diag}\left(p_{i} \circ\left(1-p_{i}\right)\right) \boldsymbol{Z}_{i}+\Gamma^{-1}\right)^{-1}, \quad p_{i}=p_{i}\left(\widehat{\mu}_{\alpha_{i}}\right)
$$

We note that for the Gaussian flexible linear mixed model in Section 6.2.4. $\widehat{\mu}_{\alpha_{i}}$ and $\widehat{\Sigma}_{\alpha_{i}}$ can be derived analytically.

The gradient $\nabla_{\theta} \ell_{i}(\theta)$ can be estimated as follows. 
- Generate $N$ samples $\alpha_{i}^{(j)} \sim \mathcal{N}\left(\widehat{\mu}_{\alpha_{i}}, \widehat{\Sigma}_{\alpha_{i}}\right), j=1, \ldots, N$.

- Compute the weights

$$
w_{i}^{(j)}=\exp \left(f\left(\alpha_{i}^{(j)}\right)+\frac{1}{2}\left(\alpha_{i}^{(j)}-\widehat{\mu}_{\alpha_{i}}\right)^{\top} \widehat{\Sigma}_{\alpha_{i}}^{-1}\left(\alpha_{i}^{(j)}-\widehat{\mu}_{\alpha_{i}}\right)\right)
$$

and $W_{i}^{(j)}=w_{i}^{(j)} / \sum_{k=1}^{N} w_{i}^{(k)}$.

- Compute the estimate

$$
\widehat{\nabla_{\theta} \ell_{i}(\theta)}=\sum_{j=1}^{N} \nabla_{\theta}\left\{\log p\left(\alpha_{i}^{(j)} \mid \Gamma\right)+\sum_{t=1}^{T_{i}}\left[y_{i t} z_{i t}^{\top}\left(\beta+\alpha_{i}^{(j)}\right)-\log \left(1+e^{z_{i t}^{\top}\left(\beta+\alpha_{i}^{(j)}\right)}\right)\right]\right\} W_{i}^{(j)} .
$$

Because the parameters $\Gamma_{j}$ are positive, a suitable transformation is needed before applying the Gaussian VB approximation. We use the transformation $\theta_{\Gamma_{j}}=\log \left(\Gamma_{j}\right)$, $j=0, \ldots, M$. Let $\widetilde{\theta}=\left(w, \beta, \theta_{\Gamma_{0}}, \ldots, \theta_{\Gamma_{m}}\right)$, then,

$$
\theta=\theta(\widetilde{\theta})=\left(w, \beta, \exp \left(\theta_{\Gamma_{0}}\right), \ldots, \exp \left(\theta_{\Gamma_{m}}\right)\right) .
$$

The posterior distribution of $\widetilde{\theta}$ is

$$
\left.p(\widetilde{\theta} \mid D) \propto\left|\frac{\partial \theta(\widetilde{\theta})}{\partial \widetilde{\theta}}\right| p(\theta(\widetilde{\theta})) p(\theta(\widetilde{\theta}) \mid D)=\exp \left(\theta_{\Gamma_{0}}+\ldots+\theta_{\Gamma_{m}}\right)\right) p(\theta(\widetilde{\theta})) p(\theta(\widetilde{\theta}) \mid D)
$$

We then approximate $p(\widetilde{\theta} \mid D)$ by $q_{\lambda}(\widetilde{\theta})$.

\section{References}

Amari, S. (1998). Natural gradient works efficiently in learning. Neural computation, 10(2):251-276.

Andrews, D. F. and Mallows, C. L. (1974). Scale mixtures of normal distributions. Journal of the Royal Statistical Society, Series B, 36:99-102.

Baltagi (2013). Econometric Analysis of Panel Data, 5th Edition. Wiley.

Bartholomew, D. J., Knott, M., and Moustaki, I. (2011). Latent variable models and factor analysis: A unified approach, 3rd edition. John Wiley \& Sons.

Bengio, Y. (2012). Practical recommendations for gradient-based training of deep architectures. In G., M., G.B., O., and KR., M., editors, Neural Networks: Tricks of the Trade. Lecture Notes in Computer Science, volume 7700. Springer, Berlin, Heidelberg. 
Bishop, C. M. (2006). Pattern Recognition and Machine Learning. New York: Springer.

Blei, D. M., Kucukelbir, A., and McAuliffe, J. D. (2017). Variational inference: A review for statisticians. Journal of the American Statistical Association, 112(518):859-877.

Carvalho, C. M., Polson, N. G., and Scott, J. G. (2010). The horseshoe estimator for sparse signals. Biometrika, 97:465-480.

Casella, G. (2001). Empirical Bayes Gibbs sampling. Biostatistics, 2:485-500.

Chipman, H. A., George, E. I., and McCulloch, R. E. (2010). Bart: Bayesian additive regression trees. Ann. Appl. Stat., 4(1):266-298.

Cornwell, C. and Rupert, P. (1988). Efficient estimation with panel data: An empirical comparison of instrumental variable estimators. Journal of Applied Econometrics, 3(2):149-155.

Fan, K., Wang, Z., Beck, J., Kwok, J., and Heller, K. (2015). Fast second-order stochastic backpropagation for variational inference. In Cortes, C., Lawrence, N. D., Lee, D. D., Sugiyama, M., and Garnett, R., editors, Advances in Neural Information Processing Systems 28, pages 1387-1395. Curran Associates, Inc.

Geweke, J. and Zhou, G. (1996). Measuring the pricing error of the arbitrage pricing theory. Review of Financial Studies, 9(2):557-587.

Glorot, X. and Bengio, Y. (2010). Understanding the difficulty of training deep feedforward neural networks. In Proceedings of the Thirteenth International Conference on Artificial Intelligence and Statistics, PMLR, volume 9, pages 249-256.

Goodfellow, I., Bengio, Y., and Courville, A. (2016). Deep Learning. MIT Press.

Gunawan, D., Tran, M.-N., and Kohn, R. (2017). Fast inference for intractable likelihood problems using variational Bayes. Technical report. arXiv:1705.06679.

Han, S., Liao, X., Dunson, D. B., and Carin, L. C. (2016). Variational Gaussian copula inference. In Gretton, A. and Robert, C. C., editors, Proceedings of the 19th International Conference on Artificial Intelligence and Statistics, volume 51, pages 829-838, Cadiz, Spain. JMLR Workshop and Conference Proceedings.

Hoffman, M. D., Blei, D. M., Wang, C., and Paisley, J. (2013). Stochastic variational inference. Journal of Machine Learning Research, 14:1303-1347.

Honkela, A., Raiko, T., Kuusela, M., Tornio, M., and Karhunen, J. (2010). Approximate Riemannian conjugate gradient learning for fixed-form variational Bayes. Journal of Machine Learning Research, 11:3235-3268. 
Jank, W. (2011). Business Analytics for Managers. Springer-Verlag New York.

Kingma, D. P. and Welling, M. (2013). Auto-encoding variational Bayes. Technical report. arXiv:1312.6114v10.

Kucukelbir, A., Tran, D., Ranganath, R., Gelman, A., and Blei, D. M. (2017). Automatic differentiation variational inference. Journal of Machine Learning Research, $18(14): 1-45$.

Kyung, M., Gill, J., Ghosh, M., and Casella, G. (2010). Penalized regression, standard errors and Bayesian Lassos. Bayesian Statistics, 5:369-412.

Lai, T. L., Shih, M.-C., and Wong, S. P. (2006). A new approach to modeling covariate effects and individualization in population pharmacokinetics-pharmacodynamics. Journal of Pharmacokinetics and Pharmacodynamics, 33(1):49-74.

Leng, C., Tran, M.-N., and Nott, D. J. (2014). Bayesian adaptive lasso. The Annals of the Institute of Statistical Mathematics, 66:221-44.

Martens, J. (2010). Deep learning via Hessian-free optimization. In 27th International Conference on Machine Learning, Haifa, Israel.

Martens, J. (2014). New insights and perspectives on the natural gradient method. Technical report. arXiv:1412.1193.

Ong, V. M.-H., Nott, D. J., and Smith, M. S. (2017a). Gaussian variational approximation with factor covariance structure. Journal of Computational and Graphical Statistics, To Appear.

Ong, V. M.-H., Nott, D. J., Tran, M.-N., Sisson, S., and Drovandi, C. (2018). Variational Bayes with synthetic likelihood. Statistics and Computing, To appear.

Ong, V. M.-H., Nott, D. J., Tran, M.-N., Sisson, S. A., and Drovandi, C. C. (2017b). Likelihood-free inference in high dimensions with synthetic likelihood. Technical report, Queensland University of Tehcnology, https://eprints.qut.edu.au/ $112213 /$.

Pascanu, R. and Bengio, Y. (2014). Revisiting natural gradient for deep networks. Technical report. arXiv:1301.3584v7.

Polson, N. G. and Sokolov, V. O. (2017). Deep learning: A Bayesian perspective. Technical report. arXiv:1706.00473v1.

Polyak, B. T. (1964). Some methods of speeding up the convergence of iteration methods. USSR Computational Mathematics and Mathematical Physics, 4(5). 
Ranganath, R., Wang, C., Blei, D. M., and Xing, E. P. (2013). An adaptive learning rate for stochastic variational inference. In Proceedings of the 30th International Conference on Machine Learning (ICML), pages 298-306.

Rao, C. R. (1945). Information and accuracy attainable in the estimation of statistical parameters. Bull. Calcutta. Math. Soc, 37:81-91.

Regier, J., Jordan, M. I., and McAuliffe, J. (2017). Fast black-box variational inference through stochastic trust-region optimization. arXiv preprint arXiv:1706.02375.

Rezende, D. J., Mohamed, S., and Wierstra, D. (2014). Stochastic backpropagation and approximate inference in deep generative models. In Proceedings of the 31st International Conference on Machine Learning (ICML-14), pages 1278-1286.

Robbins, H. and Monro, S. (1951). A stochastic approximation method. Annals of Mathematical Statistics, 22:400-407.

Roeder, G., Wu, Y., and Duvenaud, D. (2017). Sticking the landing: Simple, lower-variance gradient estimators for variational inference. arXiv preprint arXiv:1703.09194.

Salimans, T. and Knowles, D. A. (2013). Fixed-form variational posterior approximation through stochastic linear regression. Bayesian Analysis, 8(4):741-908.

Sato, M. (2001). Online model selection based on the variational Bayes. Neural Computation, 13(7):1649-1681.

Schmidhuber, J. (2015). Deep learning in neural networks: An overview. Neural Networks, 61:85- 117 .

Stoer, J. (1983). Solution of large linear systems of equations by conjugate gradient type methods. In Bachem, A., Korte, B., and Grötschel, M., editors, Mathematical Programming The State of the Art: Bonn 1982, pages 540-565. Springer Berlin Heidelberg, Berlin, Heidelberg.

Tan, L. S. L. and Nott, D. J. (2017). Gaussian variational approximation with sparse precision matrices. Statisics and Computing, To Appear.

Titsias, M. and Lázaro-Gredilla, M. (2014). Doubly stochastic variational Bayes for non-conjugate inference. In Proceedings of the 31st International Conference on Machine Learning (ICML-14), pages 1971-1979.

Tran, M., Nott, D., and Kohn, R. (2017). Variational Bayes with intractable likelihood. Journal of Computational and Graphical Statistics, 26(4):873-882.

Tran, M.-N., Kohn, R., Quiroz, M., and Villani, M. (2016a). Block-wise pseudomarginal Metropolis-Hastings. Technical report. arXiv:1603.02485. 
Tran, M.-N., Nott, D. J., Kuk, A. Y., and Kohn, R. (2016b). Parallel variational Bayes for large datasets with an application to generalized linear mixed models. Journal of Computational and Graphical Statistics, 26:626-646.

Trippe, B. L. and Turner, R. E. (2018). Overpruning in variational Bayesian neural networks. arXiv:1801.06230v1.

Yuan, M. and Lin, Y. (2006). Model selection and estimation in regression with grouped variables. Journal of the Royal Statistical Society, Series B, 68:49-67.

Zeiler, M. D. (2012). Adadelta: An adaptive learning rate method. Technical report. arXiv:1212.5701. 